\title{
Using electron scattering superscaling to predict charge-changing neutrino cross sections in nuclei
}

\author{
J. E. Amaro, ${ }^{1}$ M. B. Barbaro, ${ }^{2}$ J. A. Caballero, ${ }^{3}$ T. W. Donnelly, ${ }^{4}$ A. Molinari, ${ }^{2}$ and I. Sick ${ }^{5, *}$ \\ ${ }^{1}$ Departamento de Física Moderna, Universidad de Granada, E-18071 Granada, Spain \\ ${ }^{2}$ Dipartimento di Fisica Teorica, Università di Torino, and INFN, Sezione di Torino, Via P. Giuria 1, I-10125 Torino, Italy \\ ${ }^{3}$ Departamento de Física Atómica, Molecular y Nuclear, Universidad de Sevilla, Apdo. 1065, E-41080 Sevilla, Spain \\ ${ }^{4}$ Center for Theoretical Physics, Laboratory for Nuclear Science, and Department of Physics, Massachusetts Institute of Technology, \\ Cambridge, Massachusetts 02139, USA \\ ${ }^{5}$ Departement für Physik und Astronomie, Universität Basel, CH-4056 Basel, Switzerland
}

(Received 1 October 2004; published 27 January 2005)

\begin{abstract}
Superscaling analyses of few-GeV inclusive electron scattering from nuclei are extended to include not only quasielastic processes, but also the region where $\Delta$ excitation dominates. With reasonable assumptions about the basic nuclear scaling function extracted from data and information from other studies of the relative roles played by correlation and meson-exchange-current effects, it is shown that the residual strength in the resonance region can be accounted for through an extended scaling analysis. One observes scaling upon assuming that the elementary cross section by which one divides the residual to obtain a new scaling function is dominated by the $N \rightarrow \Delta$ transition and employing a new scaling variable suited to the resonance region. This yields a good representation of the electromagnetic response in both the quasielastic and $\Delta$ regions. The scaling approach is then inverted and predictions are made for charge-changing neutrino reactions at energies of a few $\mathrm{GeV}$, with focus placed on nuclei that are relevant to neutrino oscillation measurements. For this, a relativistic treatment of the required weak interaction vector and axial-vector currents for both quasielastic and $\Delta$-excitation processes is presented.
\end{abstract}

DOI: 10.1103/PhysRevC.71.015501

PACS number(s): 25.30.Pt, 23.40.Bw, 24.10.Jv

\section{INTRODUCTION}

In recent studies of inclusive electron scattering at intermediate to high energies from nuclei we explored various aspects of scaling and superscaling [1-7]. The general procedure used in such analyses is to divide the experimental inclusive cross section by an appropriate single-nucleon cross section, having contributions from $Z$ protons and $N$ neutrons with their corresponding electromagnetic form factors, to obtain a reduced cross section. Here the word "appropriate" entails two things. First, the usual analysis in the region of the quasielastic $(\mathrm{QE})$ peak assumes that the dominant process is elastic scattering from nucleons in the nuclear ground state followed by quasifree ejection of the nucleons from the nucleus, and hence the appropriate single-nucleon form factors are the elastic ones. Second, the nucleons in the nuclear ground state are moving (Fermi motion) and accordingly the single-nucleon cross section used must take this into account. Once one has the reduced cross section it can be plotted against one or more appropriately chosen variables; if the results do not depend on some of these variables and a universal behavior is found, one says that the results scale. Specifically, when the reduced cross section is plotted against a well-chosen scaling variable (see below) and no dependence on the momentum transfer $q$ is observed, one says that one has scaling of the first kind. When no dependence occurs on the momentum scale that characterizes specific nuclei (essentially the Fermi momentum $k_{F}$ of a given nuclear species), one says that one has scaling of

* To contact collaboration, send e-mail to: Ingo.Sick@unibas.ch. the second kind. If both types of scaling behavior are found, one says that superscaling occurs.

At sufficiently high energies we have seen both types of scaling behavior. For specific nuclei one observes quite good first-kind scaling at excitation energies below the QE peak, namely, in the so-called scaling region. This is the familiar $y$-scaling behavior. At energies above the peak, where nucleon resonances (especially the $\Delta$ ) are important, this type of scaling is broken for the total reduced cross section. On the other hand, from what data we have where longitudinaltransverse separations have been made, we know that these scaling violations apparently reside in the transverse response, but not in the longitudinal. The latter appears to superscale. In fact, this is not unexpected, since we know that there are contributions that do not scale arising from meson-exchange currents (MECs) plus the correlation effects ${ }^{1}$ required by gauge invariance which must be considered together with the MEC [8-10] and from inelastic scattering from the nucleons [6]. As discussed below, it is important to observe that MEC and inelastic contributions are predominantly transverse in the kinematic regions of interest in the present work. Note that scaling of the second kind works very well in the scaling region and, even in the resonance region, it is only violated at roughly the $20 \%$ level.

\footnotetext{
${ }^{1}$ Note that such correlation effects are not the only ones. In particular, even in a factorized approach of the type presented here, there are both mean-field and short-range correlations in the initial state which are embodied in a nuclear spectral function and which lead to the scaling function deduced from data, as discussed later.
} 
Using these recent studies as a basis we now extend our analysis to encompass both the $\mathrm{QE}$ and $\Delta$ regions. Our approach is the following: taking all of the high-quality data for two specific nuclei of relevance to our later discussions, namely for ${ }^{12} \mathrm{C}$ and ${ }^{16} \mathrm{O}$, we proceed as outlined above and begin by performing a comprehensive scaling analysis in the $\mathrm{QE}$ region. Using our knowledge of the experimentally determined longitudinal superscaling function as the starting point, we work backward and predict the transverse response one would obtain strictly from the contributions that are present in that function. In other words, we reconstruct the part of the transverse cross section that has neither MEC effects nor inelasticity built into it. The net inclusive cross section so obtained is then, in effect, the QE contribution, except for corrections arising from MEC effects and their associated correlations. The next step is to subtract this from the data. What is left should now be dominated by the inelasticity; and, in particular, when not too far above the QE peak region, we expect the $\Delta$ resonance to be the most important contribution.

From other studies, we expect that a similar procedure can now be followed for the subtracted results. We again reduce the leftover cross section by dividing by the appropriate single-nucleon cross section, now for the $N \rightarrow \Delta$ transition, and display the result versus a new scaling variable in which the kinematics of resonance electro-production are respected. As discussed later, the results scale quite well, suggesting that this procedure has indeed identified the dominant contributions not only in the QE region, but also in the $\Delta$ region. We check our analysis by assembling all of the pieces obtained via the scaling procedures to produce a total inclusive cross section which can be compared with data. Overall the answers are very encouraging, and only for specific kinematics do we see deviations as large as 10-20\%. We believe that effects from MECs and their associated correlations (which are not incorporated using this approach) are probably responsible for these residuals.

Having met with success in extending the scaling and superscaling analyses from the scaling region, through the $\mathrm{QE}$ peak region, and now into the $\Delta$ region, we are in a position to take a step in a new direction. Since we have the scaling functions and can be sure that, upon being multiplied by the electromagnetic $N \rightarrow N$ and $N \rightarrow \Delta$ single-nucleon cross sections, the total nuclear electromagnetic cross section is quite well reproduced, we can just as well multiply by the corresponding charge-changing weak interaction $N \rightarrow N$ and $N \rightarrow \Delta$ single-nucleon cross sections to obtain predictions for neutrino reactions in nuclei at similar high-energy kinematics.

Thus, the second motivation for the present investigation has been to work backward to predictions for these cross sections with the goal of providing high-quality results for use in ongoing experimental studies of neutrino oscillations at $\mathrm{GeV}$ energies [11-15]. These studies are presently being pursued in the MiniBooNE and K2K/T2K experiments [11]. Both of these and also the forthcoming MINOS, NOvA, and MINERvA [12-15] initiatives involve neutrino energies of several $\mathrm{GeV}$ where a fully relativistic treatment of the neutrino-nucleus scattering is mandatory, but hard to achieve. Targets of hydrocarbon or water are involved in the cases of MiniBooNE and $\mathrm{K} 2 \mathrm{~K} / \mathrm{T} 2 \mathrm{~K}$, and hence ${ }^{12} \mathrm{C}$ and ${ }^{16} \mathrm{O}$ are taken as the focus in the present work. For the others, iron and lead will also be considered, and in this regard we note that, to the degree that scaling of the second kind is reasonably well satisfied, one can focus on the nuclei where the most reliable electron scattering data are available and then subsequently obtain predictions for neutrino reactions not only for those nuclei, but also for a wide range of targets.

Any reliable calculation for neutrino scattering should first be tested against electron scattering data. Here, instead of using a specific model to describe inclusive electron scattering at relatively high energies in the $\mathrm{QE}$ and $\Delta$ regions, as stated above, we follow a different approach. Using as a basis the scaling behavior of the electron-nucleus cross section in both the QE and $\Delta$ peaks, we extract the scaling functions directly from experimental data and use them to predict the neutrinonucleus cross section.

This strategy is motivated by the fact that while relativistic modeling [16-25] of the nuclear dynamics in studies of highenergy inclusive lepton scattering is expected to be capable of getting the basic size and shape of the cross section, so far it has not been capable of accounting for important details of the response. Specifically, such modeling has been able to provide a reasonable representation of the $e A$ inclusive cross section. Typically at high energies the cross sections obtained using wide classes of models, including those with relativistic meanfield dynamics and random-phase approximation (RPA)-type correlations included, are seen to be very similar to the results found using the relativistic Fermi gas (RFG) model, accordingly we will also compare the results obtained using the scaling approach with those obtained using the RFG model. At the peaks of the $\mathrm{QE}$ or $\Delta$ responses, one finds that the two approaches differ by about $25 \%$ (with mean-field effects this discrepancy is reduced to perhaps 20\%); however, as we shall see later, the phenomenological scaling approach requires a long tail, which is largely absent in most modeling. A possible reason for this disagreement is the absence of classes of short-range correlation effects in most of the relativistic modeling. It goes without saying that nonrelativistic modeling is completely inadequate at the energies of interest in the present work. At intermediate energies (below those considered in this study) it is, of course, important to include effects from final-state interactions and RPA correlations (see for instance recent work reported in $[26,27]$ ) as these can be significant.

Since most semileptonic scattering processes at similar kinematics have much the same character, one should expect that failure at this level will also imply a similar level of disagreement for predictions made of neutrino reaction cross sections using the same types of modeling. Clearly, on the one hand, if all one wants is a rough estimate of neutrino reaction cross sections at similar energies, then existing relativistic modeling is probably adequate. On the other hand, if uncertainties of less than $25 \%$ are required [as, for example, when one wishes to see distortions in the energy distributions of the detected muons in $\left(v_{\mu}, \mu^{-}\right)$reactions with nuclei caused by neutrino oscillations], then one must use existing models with great caution. As we shall see below, the superscaling approach being followed in the present work appears to be capable of reducing the uncertainty to perhaps the $10 \%$ level, 
at least when one limits the focus to the QE region and the region up to inelasticities where the $\Delta$ contribution reaches its maximum.

The paper is organized the following way. In Sec. II we begin with a brief discussion of kinematics, since we will be interested not only in electron-neutrino-induced reactions, where the lepton masses can safely be ignored, but also in muon-neutrino-induced reactions where the energies, although relatively high, are not high enough to safely ignore the muon mass. In Sec. III we present a summary of the formalism needed in studies of scaling and superscaling, both for the $\mathrm{QE}$ and $\Delta$ regions. There we give the results of our analysis of inclusive high-energy inelastic electron scattering data for carbon and oxygen, using the procedures outlined above, and thereby validate the scaling functions we use in the rest of the paper. In Sec. IV we turn to the second theme of the paper and discuss charge-changing neutrino reactions with nuclei. We begin by presenting the basic formalism required in treating electroweak processes, followed by development of the single-nucleon responses in the QE region (Sec. IV A) and in the $\Delta$ region (Sec. IV B), now of course with both vector and axial-vector $N \rightarrow N$ and $N \rightarrow \Delta$ currents. Section IV C then contains a discussion of the formalism involved in obtaining the cross sections and response functions. Once these developments are in hand, we proceed in Sec. V with a presentation of our predictions for charge-changing neutrino reactions with nuclei. Finally, in Sec. VI we summarize our work and present our conclusions.

\section{LEPTON SCATTERING KINEMATICS}

In this section we begin with a brief discussion of the kinematics involved in studies of lepton scattering from nuclei including electron scattering and the subject of Sec. IV, charge-changing neutrino reactions. We start with a general scattering problem in which an incident beam of leptons with 4-momentum $K^{\mu}=(\epsilon, \mathbf{k})$ scatters and a lepton with 4-momentum $K^{\prime \mu}=\left(\epsilon^{\prime}, \mathbf{k}^{\prime}\right)$ emerges. In general, one has

$$
\begin{aligned}
\epsilon & =\sqrt{m^{2}+k^{2}}, \\
\epsilon^{\prime} & =\sqrt{m^{\prime 2}+k^{\prime 2}},
\end{aligned}
$$

where $m$ and $m^{\prime}$ are the masses of the incident and outgoing leptons, respectively. Clearly, for electron scattering $m=$ $m^{\prime}=m_{e}$ (usually, but not always, this can be taken to be zero) and for electron-neutrino-induced charge-changing neutrino reactions $m=m_{v e} \cong 0$, whereas $m^{\prime}=m_{e}$ (again, essentially zero). The difficult case is for muon-neutrino-induced chargechanging neutrino reactions where $m=m_{\nu \mu} \cong 0$, whereas $m^{\prime}=m_{\mu}$; the last is clearly not negligible for the kinematics of interest in the present work.

As usual, one has a 4-momentum transfer $Q^{\mu}=(\omega, \mathbf{q})$ with

$$
\begin{aligned}
& \omega=\epsilon-\epsilon^{\prime}, \\
& \mathbf{q}=\mathbf{k}-\mathbf{k}^{\prime},
\end{aligned}
$$

the energy transfer and 3-momentum transfer, respectively. The momentum transfer is spacelike: $-Q^{2}=q^{2}-\omega^{2}>0$.
For convenience we define an average leptonic mass as

$$
M \equiv \sqrt{\frac{1}{2}\left(m^{2}+m^{\prime 2}\right)} \geqslant 0,
$$

and given an excitation from target rest mass $M_{i}$ to some final rest mass $M_{f} \geqslant M_{i}$ (that is, the final hadronic rest frame total energy is $W=M_{f}$ ), we define a sort of excitation energy as

$$
\omega_{0} \equiv \frac{1}{2 M_{i}}\left(M_{f}^{2}-M_{i}^{2}\right) \geqslant 0
$$

Then from energy-momentum conservation one has

$$
\omega=\omega_{0}+\frac{\left|Q^{2}\right|}{2 M_{i}} .
$$

Solving Eqs. (3) and (7) together produces expressions for the scattered lepton's energy and 3-momentum. Defining

$$
\begin{aligned}
& \epsilon_{1} \equiv \sqrt{M_{i}^{2}+2 M_{i} \epsilon+m^{2}+k^{2} \sin ^{2} \theta}, \\
& \epsilon_{2} \equiv \sqrt{M_{i}\left(\epsilon-\omega_{0}\right)+M^{2}},
\end{aligned}
$$

where $\theta$ is the lepton scattering angle (the angle between $\mathbf{k}$ and $\mathbf{k}^{\prime}$ ), it can be shown that

$$
\begin{aligned}
& k^{\prime}=\frac{1}{\epsilon_{1}^{2}}\left[\epsilon_{2}^{2}(k \cos \theta)+\left(M_{i}+\epsilon\right) \sqrt{\epsilon_{2}^{4}-m^{\prime 2} \epsilon_{1}^{2}}\right], \\
& \epsilon^{\prime}=\frac{1}{\epsilon_{1}^{2}}\left[\epsilon_{2}^{2}\left(M_{i}+\epsilon\right)+(k \cos \theta) \sqrt{\epsilon_{2}^{4}-m^{\prime 2} \epsilon_{1}^{2}}\right],
\end{aligned}
$$

where for the results to be real for all scattering angles the beam energy must be greater than $\epsilon_{\min }$, where

$$
\epsilon_{\min }=m^{\prime}+\omega_{0}+\frac{m^{\prime} \omega_{0}+\left(m^{\prime 2}-m^{2}\right) / 2}{M_{i}-m^{\prime}} .
$$

Hence, for a given excitation energy $\omega_{0}$ and for given beam energy and scattering angle, the quantities $\epsilon_{1,2}$ can be computed and through them the final lepton's energy and 3 -momentum are fixed. Clearly the 4-momentum transfer is then given as well.

\section{SCALING AND SUPERSCALING}

\section{A. Scaling in the quasielastic peak region}

In this subsection we briefly review the structure of the nuclear responses in the region of the quasielastic peak (QEP). We begin with the basic relativistic Fermi gas model that has been used to motivate scaling and superscaling behavior in this region of kinematics [1-4]. Here a single parameter characterizes the dynamics, namely, the Fermi momentum $k_{F}$. In the present work our goal is to use the electron scattering cross sections as input, perform a scaling analysis, and arrive at predictions for the charge-changing neutrino cross sections. Accordingly the focus is placed on kinematic regimes where the cross sections (induced by electrons or neutrinos) are substantial, and from past work it is known that under such circumstances it is a good approximation to work only to leading order in an expansion in $\eta_{F} \equiv k_{F} / m_{N}$. Also $\xi_{F} \equiv \sqrt{1+\eta_{F}^{2}}-1 \cong \eta_{F}^{2} / 2$ is small. 
The leading-order QE responses (denoted by subscript zero) may be written, in the non-Pauli-blocked domain, in the following form [1]:

$$
\begin{gathered}
R_{L}^{\mathrm{QE}}(\kappa, \lambda)_{0}=\Lambda_{0} \frac{\kappa^{2}}{\tau}\left[(1+\tau) W_{2}(\tau)-W_{1}(\tau)\right] f_{\mathrm{RFG}}(\psi), \\
R_{T}^{\mathrm{QE}}(\kappa, \lambda)_{0}=\Lambda_{0}\left[2 W_{1}(\tau)\right] \times f_{\mathrm{RFG}}(\psi),
\end{gathered}
$$

with

$$
\Lambda_{0} \equiv \frac{\mathcal{N} \xi_{F}}{m_{N} \kappa \eta_{F}^{3}} \cong \frac{\mathcal{N}}{2 \kappa k_{F}},
$$

and $W_{1}, W_{2}$ the structure functions for elastic scattering. As usual the proton $(\mathcal{N}=Z)$ and neutron $(\mathcal{N}=N)$ contributions should be separately computed with the appropriate form factors and added together. The latter are linked to the Sachs form factors through the well-known relations

$$
\begin{gathered}
(1+\tau) W_{2}(\tau)-W_{1}(\tau)=G_{E}^{2}(\tau), \\
2 W_{1}(\tau)=2 \tau G_{M}^{2}(\tau) .
\end{gathered}
$$

As usual, we employ dimensionless variables $\lambda \equiv \omega / 2 m_{N}$, $\kappa \equiv q / 2 m_{N}$, and $\tau \equiv\left|Q^{2}\right| / 4 m_{N}^{2}=\kappa^{2}-\lambda^{2}$. The RFG has the following universal form for the superscaling function:

$$
f_{\mathrm{RFG}}(\psi)=\frac{3}{4}\left(1-\psi^{2}\right) \theta\left(1-\psi^{2}\right),
$$

that is, when plotted against the scaling variable $\psi$,

$$
\psi \equiv \frac{1}{\sqrt{\xi_{F}}} \frac{\lambda-\tau}{\sqrt{(1+\lambda) \tau+\kappa \sqrt{\tau(1+\tau)}}},
$$

a universal behavior is obtained with no dependence left either on momentum transfer (scaling of the first kind) or on nuclear species via $k_{F}$ (scaling of the second kind).

In studies of electron scattering scaling, one usually includes a small energy shift by replacing $\omega$ by $\omega-E_{\text {shift }}$ in order to force the maximum of the $\mathrm{QE}$ response to occur for $\psi^{\prime}=0$ (see, for example [3-5,28]). This is equivalent to taking $\lambda \rightarrow \lambda^{\prime}=\lambda-\lambda_{\text {shift }}$ with $\lambda_{\text {shift }}=E_{\text {shift }} / 2 m_{N}$ and correspondingly $\tau \rightarrow \tau^{\prime}=\kappa^{2}-\lambda^{\prime 2}$ in Eq. (19). Often we shall use the pair of variables $\left(\kappa, \psi^{\prime}\right)$ in place of the original pair $(q, \omega)$ to characterize the inclusive scattering responses - clearly they are functionally related by the above equations.

Using the guidance provided by the RFG, the procedure to adopt in order to get the experimental scaling function $F^{\mathrm{QE}}\left(\kappa, \psi^{\prime}\right)$ in the $\mathrm{QE}$ domain is then clear: Simply divide the experimental $\mathrm{QE}$ cross section by

$$
S^{\mathrm{QE}} \equiv \sigma_{M}\left[v_{L} G_{L}^{\mathrm{QE}}+v_{T} G_{T}^{\mathrm{QE}}\right],
$$

where $\sigma_{M}$ is the Mott cross section, $v_{L}, v_{T}$ are the kinematic factors defined below in Eqs. (74) and (75), and the functions $G_{L, T}^{\mathrm{QE}}$ are [2-6]

$$
\begin{aligned}
G_{L}^{\mathrm{QE}} & =\frac{\kappa}{2 \tau}\left[Z G_{E, p}^{2}+N G_{E, n}^{2}\right]+\mathcal{O}\left(\eta_{F}^{2}\right), \\
G_{T}^{\mathrm{QE}} & =\frac{\tau}{\kappa}\left[Z G_{M, p}^{2}+N G_{M, n}^{2}\right]+\mathcal{O}\left(\eta_{F}^{2}\right) .
\end{aligned}
$$

The factors involving $\kappa$ and $\tau$ in Eqs. (21) and (22) arise partly from the Jacobian of the transformation from $\lambda$ to $\psi$ [7] and partly from the explicit calculation leading to
Eqs. (13) and (14). Finally, as in past discussions of scaling of the second kind, one multiplies $F^{\mathrm{QE}}\left(\kappa, \psi^{\prime}\right)$ by $k_{F}$ to obtain the superscaling function $f^{\mathrm{QE}}\left(\kappa, \psi^{\prime}\right)$.

The nuclear response functions all have the general structure

$$
[R]^{\mathrm{QE}}=\frac{1}{k_{F}} f^{\mathrm{QE}}\left(\kappa, \psi^{\prime}\right) \frac{\mathcal{N}}{2 \kappa}[R]^{\mathrm{s.n} .},
$$

where $\mathcal{N}$ is the appropriate nucleon number. In particular, as stated above, one copy of this expression with proton form factors and $\mathcal{N}=Z$ should be added to another with neutron form factors and $\mathcal{N}=N$ for electron scattering. Here $[R]^{\text {s.n. }}$ is the corresponding single-nucleon response.

In previous work [2-4] we have shown that scaling works quite well for all nuclei and for energy loss $\omega<\omega_{\mathrm{QEP}}$, where $\omega_{\text {QEP }}$ corresponds to the maximum of the quasielastic peak; the scaling function $F^{\mathrm{QE}}\left(\kappa, \psi^{\prime}\right)$ is indeed largely independent of the momentum transfer as long as $q$ is of the order of $2 k_{F}$ or larger. Deviations from scaling, which mainly occur at larger energy loss, are related to contributions beyond quasielastic scattering such as those from meson exchange currents and $\Delta$ excitation (see below).

The scaling behavior becomes particularly clear if one studies the experimental response separated into its longitudinal (charge) and transverse (magnetic) pieces. The nonscaling contributions mentioned above mainly occur in the transverse response. Accordingly, one finds that the experimental longitudinal responses scale much better and to much larger energy loss. The approach taken in $[3,4]$ therefore has been to use the experimental longitudinal responses to define the scaling function $f^{\mathrm{QE}}\left(\psi^{\prime}\right)$.

The total inclusive electron scattering response is then assumed to be composed of several contributions: (1) the entire longitudinal contribution appears to superscale and to be represented by the empirical scaling function $f^{\mathrm{QE}}\left(\psi^{\prime}\right)$; (2) part of the transverse response arises from quasielastic knockout of nucleons from the nucleus and is also driven by the scaling function $f^{\mathrm{QE}}\left(\psi^{\prime}\right)$; however, (3) the transverse response has additional contributions, at least from MEC effects with their associated correlations and from inelastic single-nucleon processes including the excitation of the $\Delta$. From our past work we know that typically the effects under item (3) break the scaling. The contributions from MEC effects together with their attendant correlations enter roughly at the $10 \%$ level [8-10,29-34] and, as we argue later, may be less important for charge-changing neutrino reactions than they are for electron scattering. Accordingly we shall ignore these nonscaling effects in the present work. In future work we hope to address this issue more directly with continued relativistic modeling of these contributions. Other effects can enter into the dynamics and invalidate the picture here (for example, at low $q$, the RPA correlation effects can modify the longitudinal and transverse responses in different ways because of the very different isospin character of these two channels); however, again, at the kinematics of interest in the present work these effects are thought to be relatively small.

The largest nonscaling contribution to the transverse response is then believed to be the one arising from inelastic but impulsive processes, especially via the excitation of the $\Delta$ for 


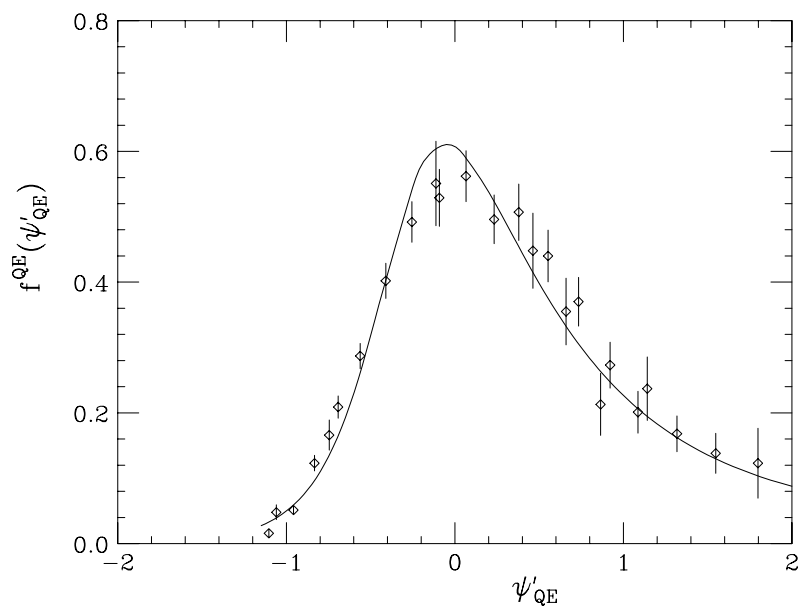

FIG. 1. Averaged experimental $f^{\mathrm{QE}}\left(\psi_{\mathrm{QE}}^{\prime}\right)$ versus $\psi_{\mathrm{QE}}^{\prime}$ in the quasielastic region together with a phenomenological parametrization of the data. The integral of the curve has been normalized to unity.

the kinematics of interest in the current study; this provides the focus for the following subsection.

In $[3,4]$ the intercomparison of the scaling functions for various nuclei has been performed in terms of the functions $f^{\mathrm{QE}}\left(\kappa, \psi^{\prime}\right)$ extracted from $F^{\mathrm{QE}}\left(\kappa, \psi^{\prime}\right)$ as discussed above. Excellent scaling of the second kind, i.e., scaling functions $f^{\mathrm{QE}}\left(\kappa, \psi^{\prime}\right)$ that closely match for different nuclei, was observed and, indeed, such second-kind scaling is actually significantly better realized than is scaling of the first kind.

The combination of scaling of the first and second kind-superscaling - allows one to determine from the data a universal scaling function $f^{\mathrm{QE}}\left(\psi^{\prime}\right)$. The scaling function (and quasielastic cross section) for individual nuclei can then be recalculated once the Fermi momentum of the nucleus is known.

Reliable separations of data into their longitudinal and transverse contributions for $A>4$ are available only for a few nuclei [35]; all of these response functions have been used to extract the "universal" quasielastic response function $f^{\mathrm{QE}}$ and to obtain a parametrization by a simple function. Figure 1 shows $f^{\mathrm{QE}}\left(\psi_{\mathrm{QE}}^{\prime}\right)$ averaged over the nuclei employed, together with the corresponding fit. ${ }^{2}$ Note that $f^{\mathrm{QE}}\left(\psi_{\mathrm{QE}}^{\prime}\right)$ has a somewhat asymmetric shape and a tail that extends toward positive values of $\psi_{\mathrm{OE}}^{\prime}$. In contrast, the RFG [see Eq. (18)] is symmetric when plotted as in the figure, is limited strictly to the region $-1 \leqslant \psi_{\mathrm{QE}}^{\prime} \leqslant+1$, and has a maximum value of $3 / 4$, whereas the empirical scaling function reaches only to about 0.6 .

One source for the difference could be typical mean-field dynamics in the initial and final nuclear states involved; however, for the kinematics of interest in the present work, both relativistic mean-field theory $[17,20,21,23]$ and relativized shell-model studies [19] appear to provide only rather modest

\footnotetext{
${ }^{2}$ In the figure and henceforth, the QE scaling variable is denoted $\psi_{\mathrm{QE}}^{\prime}$ to distinguish it from the scaling variable used in the $\Delta$ region; see later discussion.
}

differences from the RFG predictions. As stated above, nonrelativistic modeling is quite incapable for such kinematics (see, for instance, [19]).

Another source for the differences seen between the RFG or mean-field descriptions and the empirically determined scaling function arises from high-momentum components in realistic wave functions that may be large enough to produce the results shown in the figure; although much more work, especially in a relativistic context, is required to put this on a solid footing. For the present we limit our approach to phenomenology and take the scaling function from experiment. It should be emphasized at this point that much of the inability of typical modeling to account for the inclusive response at these kinematics appears to stem directly from the inability of that modeling to account for the results in the figure. We shall see later that when the empirical scaling function is used, one obtains a good representation of measured $\left(e, e^{\prime}\right)$ cross sections and, therefore, that one's confidence in proceeding to predictions for neutrino reaction cross sections must be raised.

Unseparated experimental quasielastic cross sections have been measured for several nuclei over a large range of momentum transfer (for a compilation see [4]). These data have been used to determine the values of $k_{F}$ for the nuclei considered (in [5] a table with numerical values is given) and since the evolution of $k_{F}$ with the nuclear mass number is slow, these values can easily be interpolated for any nucleus of interest.

As was pointed out earlier, the description of the experimental scaling functions involves, besides the choice of the proper $k_{F}$ that sets the overall momentum scale, the use of an energy shift $E_{\text {shift }}$ that in an average way accounts for the nucleon removal energy. This small correction has been included in $[3,4]$ in the analysis of the data, and [5] gives a table with the numerical values for various nuclei.

We should add that in order to obtain $f^{\mathrm{QE}}$ from the cross sections, we had to divide by the single-nucleon cross sections obtained from $e-p$ and $e-n$ scattering. We used the Höhler parametrization 8.2 [36]. In the range of $q$ of interest here, this parametrization agrees with more recent parametrizations fitted to a somewhat more extensive data set.

\section{B. Scaling in the region of the $\Delta$ peak}

Following the framework of $[5,37,38]$, let $m_{*}$ be the mass of a generic nucleon excitation and $\mu_{*} \equiv m_{*} / m_{N}$; hence $\mu_{*}=1$ for quasielastic scattering and $\mu_{*}=m_{\Delta} / m_{N} \equiv \mu_{\Delta}$ for electro-excitation in the $\Delta$ region. Introducing

$$
\begin{aligned}
& \beta_{*} \equiv \frac{1}{4}\left(\mu_{*}^{2}-1\right), \\
& \rho_{*} \equiv 1+\beta_{*} / \tau,
\end{aligned}
$$

we generalize the dimensionless scaling variable of the quasielastic peak as

$$
\psi_{*} \equiv\left[\frac{1}{\xi_{F}}\left(\kappa \sqrt{\rho_{*}^{2}+1 / \tau}-\lambda \rho_{*}-1\right)\right]^{1 / 2} \times\left\{\begin{array}{c}
+1 \lambda \geqslant \lambda_{*}^{0} \\
-1 \lambda \leqslant \lambda_{*}^{0}
\end{array}\right.
$$


which vanishes for

$$
\lambda=\lambda_{*}^{0}=\frac{1}{2}\left[\sqrt{\mu_{*}^{2}+4 \kappa^{2}}-1\right],
$$

or, in dimensionful variables, when

$$
\omega=\omega_{*}^{0}=\sqrt{m_{*}^{2}+q^{2}}-m_{N} .
$$

When $\mu_{*}=1$, one recovers the QE answer in Eq. (19), where $\beta_{*}=0, \rho_{*}=1$ and at the peak $\omega_{\mathrm{QE}}^{0}=\sqrt{m_{N}^{2}+q^{2}}-m_{N}$. As in the previous subsection where the QE scaling variable was discussed, here also we include the small energy shift $E_{\text {shift }}$ by making the replacement $\omega \rightarrow \omega^{\prime} \equiv \omega-E_{\text {shift }}$ with $\lambda \rightarrow \lambda^{\prime}$ and $\tau \rightarrow \tau^{\prime}$ as before. Again these replacements are made in the above equations to yield a generic shifted scaling variable $\psi_{*}^{\prime}$, and specifically for use in the $\Delta$ region the shifted scaling variable $\psi_{\Delta}^{\prime}$.

When considering the $N \rightarrow \Delta$ transition structure functions, we change notation from the general quantities $\beta_{*}, \rho_{*}$, $\psi_{*}$, etc., to $\beta_{\Delta}, \rho_{\Delta}, \psi_{\Delta}$, etc., and in addition introduce

$$
\gamma_{\Delta} \equiv \frac{1}{4}\left(\mu_{\Delta}-1\right)^{2}
$$

and

$$
\kappa_{\Delta}^{*}=\frac{1}{\mu_{\Delta}} \sqrt{\tau+\left(\tau+\beta_{\Delta}\right)^{2}},
$$

which allow us to define

$$
v_{1}^{\Delta} \equiv\left(1+\mu_{\Delta}\right)^{2}\left(\tau+\gamma_{\Delta}\right)
$$

and

$$
v_{2}^{\Delta} \equiv v_{1}^{\Delta} \frac{\tau}{\left(\mu_{\Delta} \kappa_{\Delta}^{*}\right)^{2}} .
$$

Then the $N \rightarrow \Delta$ single-baryon responses will read

$$
\begin{gathered}
w_{1}^{\Delta}(\tau)=v_{1}^{\Delta}\left[G_{M, p}^{2}(\tau)+3 G_{E, n}^{2}(\tau)\right], \\
w_{2}^{\Delta}(\tau)=v_{2}^{\Delta}\left[G_{M, p}^{2}(\tau)+3 G_{E, n}^{2}(\tau)+\frac{4 \tau}{\mu_{\Delta}^{2}} G_{C, \Delta}^{2}(\tau)\right],
\end{gathered}
$$

where the magnetic, electric, and Coulomb form factors (following [38]) are taken to be

$$
\begin{aligned}
G_{M, p}(\tau) & =2.97 g_{\Delta}\left(Q^{2}\right), \\
G_{E, n}(\tau) & =-0.03 g_{\Delta}\left(Q^{2}\right), \\
G_{C, \Delta}(\tau) & =-0.15 G_{M, p}(\tau),
\end{aligned}
$$

with

$$
g_{\Delta}\left(Q^{2}\right) \equiv \frac{G_{E, p}(\tau)}{\sqrt{1+\tau}}
$$

and

$$
G_{E, p}(\tau)=\frac{1}{[1+4.97 \tau]^{2}},
$$

namely the dipole parametrization of the proton (elastic) electric form factor (see also Sec. IV C).

As for the quasielastic region, in the $\Delta$ domain we ignore terms of order $\eta_{F}^{2}$. In this approximation (as above, denoted by the subscript 0) the RFG longitudinal and transverse $N \rightarrow \Delta$ responses will read

$$
R_{L}^{\Delta}(\kappa, \lambda)_{0}=\frac{1}{2} \Lambda_{0} \frac{\kappa^{2}}{\tau}\left[\left(1+\tau \rho_{\Delta}^{2}\right) w_{2}^{\Delta}(\tau)-w_{1}^{\Delta}(\tau)\right] f_{\mathrm{RFG}}\left(\psi_{\Delta}\right),
$$

$$
R_{T}^{\Delta}(\kappa, \lambda)_{0}=\frac{1}{2} \Lambda_{0}\left[2 w_{1}^{\Delta}(\tau)\right] \times f_{\mathrm{RFG}}\left(\psi_{\Delta}\right),
$$

where $\Lambda_{0}$ is given in Eq. (15). As usual, for electron scattering one should add the contribution obtained from Eqs. (40) and (41) computed with $\mathcal{N}=Z$ and the $p \rightarrow \Delta^{+}$structure functions to the one where Eqs. (40) and (41) are computed with $\mathcal{N}=N$ and the $n \rightarrow \Delta^{0}$ responses. Since these processes are purely isovector, clearly this is equivalent to using $\mathcal{N}=A$ with one set of the structure functions.

Again, using the guidance provided by the RFG, this procedure is easily generalized to the experimental response in the $\Delta$ region. Here, as long as density-dependent corrections (i.e., the corrections that go as $\eta_{F}^{2}$ ) are ignored as they were above, one should divide the experimental inclusive electro-excitation cross section in the $\Delta$ region by

$$
S^{\Delta} \equiv \sigma_{M}\left[v_{L} G_{L}^{\Delta}+v_{T} G_{T}^{\Delta}\right]
$$

to get the scaling function $F^{\Delta}(\kappa, \lambda)$. By comparing Eqs. (40) and (41) with Eqs. (13) and (14) one gets for the functions $G_{L, T}^{\Delta}$ the expressions

$$
\begin{gathered}
G_{L}^{\Delta}=\frac{\kappa}{2 \tau}\left[\mathcal{N}\left\{\left(1+\tau \rho_{\Delta}^{2}\right) w_{2}^{\Delta}(\tau)-w_{1}^{\Delta}(\tau)\right\}\right]+\mathcal{O}\left(\eta_{F}^{2}\right), \\
G_{T}^{\Delta}=\frac{1}{\kappa}\left[\mathcal{N}\left\{w_{1}^{\Delta}(\tau)\right\}\right]+\mathcal{O}\left(\eta_{F}^{2}\right) .
\end{gathered}
$$

Note that in Eq. (44) one has $w_{1}^{\Delta}(\tau)$, whereas in Eq. (22) the factor $\tau$ in $W_{1}(\tau)=\tau G_{M}^{2}(\tau)$ has been taken out in front. As before, one should take the results for reactions with protons with the appropriate form factors and with $\mathcal{N}=Z$ and add them to the results for reactions with neutrons again with the appropriate form factors, but now with $\mathcal{N}=N$. Finally, to get the superscaling function $f^{\Delta}(\kappa, \lambda)$ one multiplies $F^{\Delta}(\kappa, \lambda)$ by $k_{F}$.

With the formalism in hand, we now proceed in a manner that is analogous to our treatment of the data in the $\mathrm{QE}$ region; however, we are now focusing on the $\Delta$ region. To isolate the contributions in the $\Delta$ region, we subtract from the total experimental cross sections (with Coulomb distortion effects incorporated) the quasielastic cross section recalculated using the universal $f^{\mathrm{QE}}\left(\psi_{\mathrm{QE}}^{\prime}\right)$ introduced above. That is, we remove the impulsive longitudinal and transverse contributions that arise from elastic eN scattering, leaving (at least) MEC effects with their associated correlations and impulsive contributions arising from inelastic eN scattering. As discussed earlier, the MEC effects will be ignored in the present work as they are believed to provide relatively small corrections, and thus this yields, at least for $\psi_{\Delta}^{\prime}<0$, a response that is largely dominated by the $\Delta$. For energy losses beyond the maximum of the $\Delta$ peak, other resonances and, at the larger values of $q$, the tail of deep inelastic scattering contribute. As a consequence of the different $q$ dependencies of the various contributions, it has not been possible using the present approach to further 


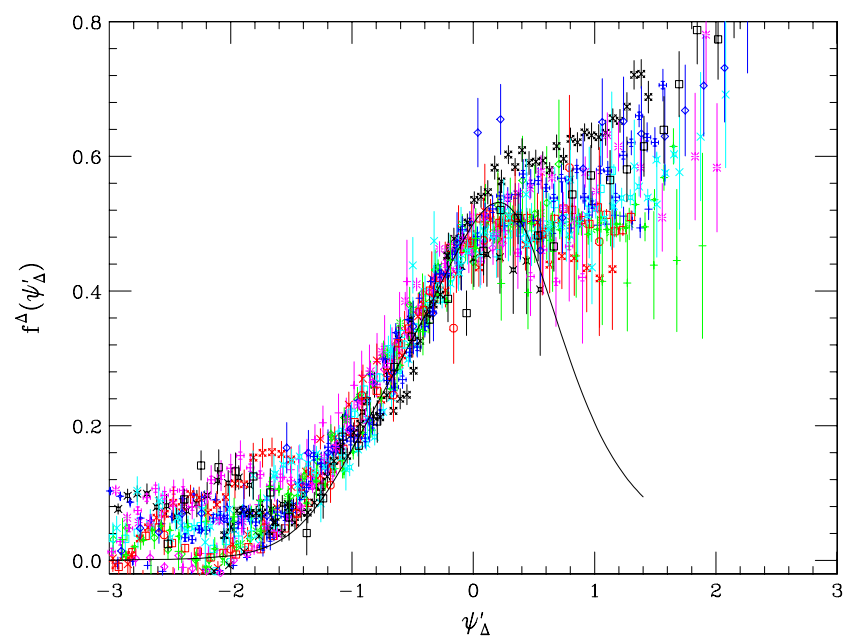

FIG. 2. (Color) Averaged experimental values of $f^{\Delta}\left(\psi_{\Delta}^{\prime}\right)$ together with a phenomenological fit (whose validity is restricted to $\psi_{\Delta}^{\prime}<0$ ).

analyze this $\psi_{\Delta}^{\prime}>0$ region. The region of validity of $f^{\Delta}\left(\psi_{\Delta}^{\prime}\right)$ therefore will be restricted to $\psi_{\Delta}^{\prime}<0$.

As shown by Eqs. (33) and (34), the determination of $f^{\Delta}(\kappa, \lambda)$ involves a division by a combination of the $M$, $E$, and $C$ contributions with their appropriate $q$ dependence. For the latter, we employ the parametrizations given in Eqs. (35)-(37) and used by Amaro et al. [38]. Obviously, the main contribution is due to the $M_{1}$ term, the $C_{0}$ and $E_{2}$ contributions to the cross sections being minor.

In Fig. 2 we show the resulting $f^{\Delta}\left(\psi_{\Delta}^{\prime}\right)$ extracted from the high-quality world data for inclusive electron scattering from ${ }^{12} \mathrm{C}$ and ${ }^{16} \mathrm{O}$ in the $\mathrm{QE}$ and $\Delta$ regions. These data span energies extending from $300 \mathrm{MeV}$ to $4 \mathrm{GeV}$ and scattering angles from 12 to 145 degrees, depending on the beam energy. For this determination the larger-angle and higher- $q$ data are of particular importance. At small angles and lower $q$, the $\Delta$ contribution is small and often not present in the available data due to limited coverage in energy loss. As for $f^{\mathrm{QE}}$, the experimental values of $f^{\Delta}$ have been parametrized by a simple analytical function. We show in Fig. 2 the averaged experimental values together with this fit. As pointed out before, the validity of the fit is restricted to $\omega$ values below the peak, i.e., $\psi_{\Delta}^{\prime}<0$.

The data appear to scale reasonably well up to the peak of the $\Delta$, namely, the point where $\psi_{\Delta}^{\prime} \cong 0$; although clearly for still higher excitation energies, the scaling is broken by processes that are not well represented via $\Delta$ dominance. There is also some excess at large negative $\psi_{\Delta}^{\prime}$ which breaks the scaling to some degree-this is thought to be due to contributions from MEC effects and their associated correlations [8-10,29-34], as discussed above. For reference we note that for electrons of $1 \mathrm{GeV}$ scattering from ${ }^{12} \mathrm{C}$, the quasielastic peak (where $\psi_{\mathrm{OE}}^{\prime} \cong 0$ ) occurs at $\psi_{\Delta}^{\prime}=-1.8$, -1.2 , and -1.0 for $\theta=45,90$, and 135 degrees, respectively. From Fig. 1 we see that $f^{\mathrm{QE}}$ peaks at roughly 0.6 and thus these nonscaling contributions typically occur at the $10-15 \%$ level in the total cross section. Below we discuss our expectations for the uncertainties incurred for our predictions

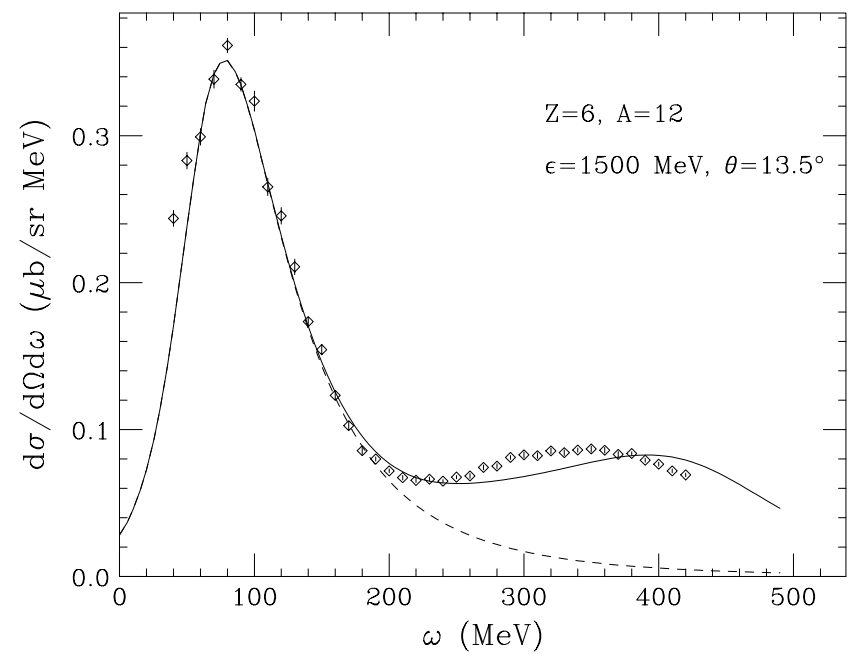

FIG. 3. Experimental $\left(e, e^{\prime}\right)$ cross section for ${ }^{12} \mathrm{C}$ at an incident electron energy of $1.5 \mathrm{GeV}$ and a scattering angle of 13.5 degrees, together with the calculated result obtained using $f^{\mathrm{QE}}$ and $f^{\Delta}$. The dashed curve is the QE contribution and the solid curve is the total including the $\Delta$.

of charge-changing neutrino reactions when we ignore such effects (see Sec. V).

In passing it is important to note that in the present study we have simply taken the residual scaling function $f^{\Delta}$ from experimental data. While similar to $f^{\mathrm{QE}}$ it differs in detail: it is somewhat lower, is shifted slightly, and is more spread out over a wider range of scaling variable. This is perhaps not unexpected, since implicit in this approach is the fact that the $\Delta$ brings with it its own width and shift. Only with a more microscopic model could one hope to be able to deconvolute these from the total response and see whether the underlying scaling function is indeed the basic $f^{\mathrm{QE}}$ deduced above. Such an approach will be pursued in the future, although it only becomes practical when the MEC contributions are under control. For the present we limit the analysis to using two different functions $f^{\mathrm{QE}}$ and $f^{\Delta}$, both deduced from phenomenological fits to electron scattering data.

With these ingredients, it is then possible to recalculate for every nucleus, incident electron energy, and scattering angle the inclusive cross section for $\omega$ below the maximum of the $\Delta$ contribution. To demonstrate this, we show in Figs. 3-5 the experimental responses together with the calculated response obtained using the parametrized $f^{\mathrm{QE}}$ and $f^{\Delta}$. In particular, we have studied the accuracy of the predicted response using $\left(e, e^{\prime}\right)$ for ${ }^{12} \mathrm{C}$ and ${ }^{16} \mathrm{O}$ and for a variety of momentum transfers, since these are the most relevant nuclei for the MiniBooNE and K2K/T2K neutrino oscillation measurements discussed in the introduction. For the data sets that do cover the $\Delta$ region, typical deviations are $10 \%$ or less.

\section{CHARGE-CHANGING NEUTRINO REACTION FORMALISM}

Among several options available (see, e.g., [20,21,23,39, 40]) we choose to write the charge-changing neutrino cross 


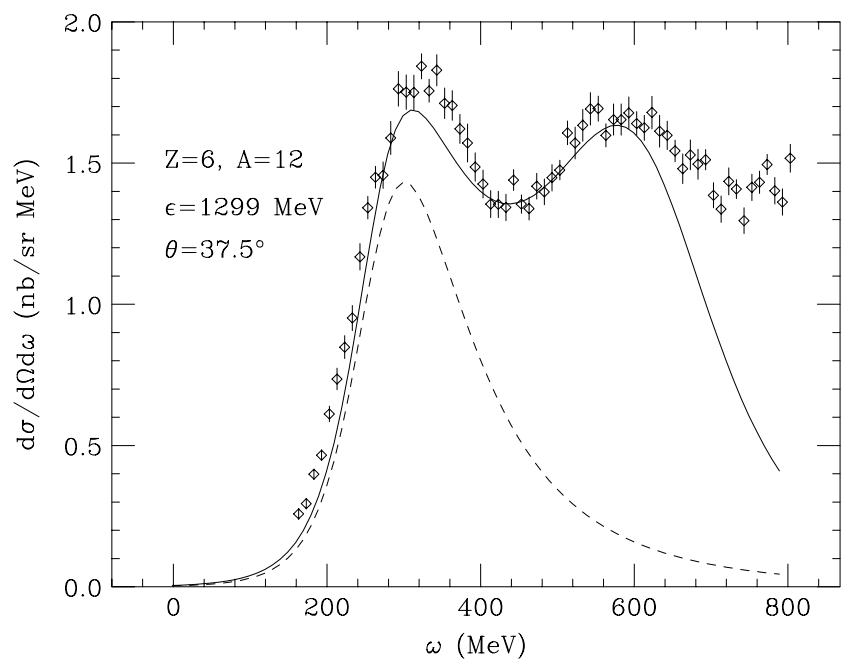

FIG. 4. As for Fig. 3, except for an electron energy of $1.3 \mathrm{GeV}$ and a scattering angle of 37.5 degrees.

section in the target laboratory frame in the form

$$
\left[\frac{d^{2} \sigma}{d \Omega d k^{\prime}}\right]_{\chi} \equiv \sigma_{0} \mathcal{F}_{\chi}^{2}
$$

where $\chi=+$ for neutrino-induced reactions (for example, $v_{l}+n \rightarrow l^{-}+p$, where $\left.l=e, \mu, \tau\right)$ and $\chi=-$ for antineutrino-induced reactions (for example, $\overline{v_{l}}+p \rightarrow l^{+}+$ $n)$. In Eq. (45)

$$
\sigma_{0} \equiv \frac{\left(G \cos \theta_{c}\right)^{2}}{2 \pi^{2}}\left[k^{\prime} \cos \tilde{\theta} / 2\right]^{2}
$$

where $G=1.16639 \times 10^{-5} \mathrm{GeV}^{-2}$ is the Fermi constant, $\theta_{c}$ is the Cabibbo angle $\left(\cos \theta_{c}=0.9741\right)$, and the generalized scattering angle $\widetilde{\theta}$ reads

$$
\tan ^{2} \tilde{\theta} / 2 \equiv \frac{\left|Q^{2}\right|}{v_{0}}
$$

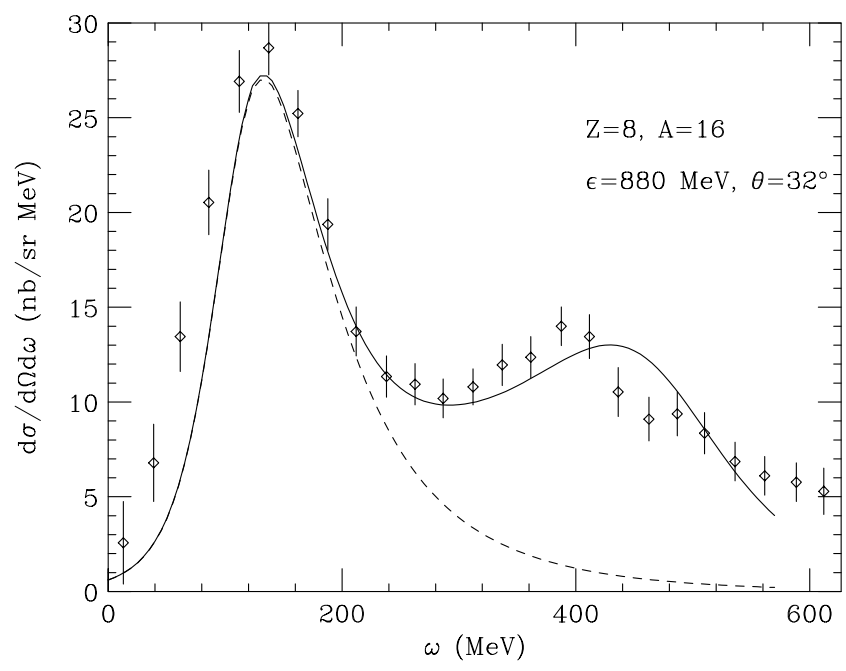

FIG. 5. Experimental $\left(e, e^{\prime}\right)$ cross section as in Figs. 3 and 4, but now for ${ }^{16} \mathrm{O}$ at an incident electron energy of $0.88 \mathrm{GeV}$ and a scattering angle of 32 degrees. with

$$
v_{0} \equiv\left(\epsilon+\epsilon^{\prime}\right)^{2}-q^{2}=4 \epsilon \epsilon^{\prime}-\left|Q^{2}\right| .
$$

Henceforth we shall assume that $m=m_{v}=0$, but will always keep $m^{\prime}$ nonzero.

One additional issue arises in computing the neutrino reaction cross sections having to do with the fact that the charged leptons in the final state are not plane waves but are influenced by the Coulomb potential provided by the nucleus. This implies that the 4-momentum of the scattered lepton, $K^{\prime \mu}=\left(\epsilon^{\prime}, \mathbf{k}^{\prime}\right)$, is the local quantity in the sense that the 3-momentum $\mathbf{k}^{\prime}$ and energy $\epsilon^{\prime}=\sqrt{m^{\prime 2}+k^{\prime 2}}$ are determined using the sequence of steps outlined in Sec. II, culminating in Eqs. (10) and (11) for these variables. However, the asymptotic energy momentum is not the same as the local quantity. Following standard procedures (see, for instance, $[21,41])$ the Coulomb interaction can be incorporated, at least approximately, by shifting from $\left(\epsilon^{\prime}, \mathbf{k}^{\prime}\right)$ to asymptotic energy-momentum $\left(\epsilon_{\infty}^{\prime}, \mathbf{k}_{\infty}^{\prime}\right)$ such that

$$
\begin{aligned}
& \mathbf{k}_{\infty}^{\prime}=D\left(k^{\prime}\right) \mathbf{k}^{\prime}, \\
& \epsilon_{\infty}^{\prime}=\sqrt{m^{\prime 2}+k^{\prime 2}},
\end{aligned}
$$

where

$$
D\left(k^{\prime}\right)=1-\chi \frac{3 Z \alpha}{2 R k^{\prime}},
$$

and $R \cong 1.2 A^{1 / 3}$ is the effective charge radius of the nucleus being studied. Thus, our procedure is to calculate the cross sections using the kinematics as discussed in Sec. II and then at the end present the results in terms of the asymptotic energies and momenta obtained in this approximate manner. The only remaining issue is that the local calculations must also be multiplied by the density-of-states factor $\left[D\left(k^{\prime}\right)\right]^{-1}$ to obtain the results we present in Sec. V.

The nuclear-structure-dependent quantity $\mathcal{F}_{\chi}^{2}$ may be written as

$$
\begin{aligned}
\mathcal{F}_{\chi}^{2}= & {\left[\widehat{V}_{C C} R_{C C}+2 \widehat{V}_{C L} R_{C L}+\widehat{V}_{L L} R_{L L}+\widehat{V}_{T} R_{T}\right] } \\
& +\chi\left[2 \widehat{V}_{T^{\prime}} R_{T^{\prime}}\right]
\end{aligned}
$$

that is, as a generalized Rosenbluth decomposition having charge-charge $(C C)$, charge-longitudinal $(C L)$, longitudinallongitudinal $(L L)$ and two types of transverse $\left(T, T^{\prime}\right)$ responses. Next we expand these response functions into their vector and axial-vector contributions according to

$$
\begin{aligned}
R_{C C} & =R_{C C}^{V V}+R_{C C}^{A A}, \\
R_{C L} & =R_{C L}^{V V}+R_{C L}^{A A}, \\
R_{L L} & =R_{L L}^{V V}+R_{L L}^{A A}, \\
R_{T} & =R_{T}^{V V}+R_{T}^{A A}, \\
R_{T^{\prime}} & =R_{T^{\prime}}^{V A} .
\end{aligned}
$$

The lepton kinematical factors are the following:

$$
\begin{aligned}
& \widehat{V}_{C C}=1-\tan ^{2} \widetilde{\theta} / 2 \cdot \delta^{2}, \\
& \widehat{V}_{C L}=v+\frac{1}{\rho^{\prime}} \tan ^{2} \tilde{\theta} / 2 \cdot \delta^{2},
\end{aligned}
$$




$$
\begin{aligned}
\widehat{V}_{L L}= & v^{2}+\tan ^{2} \tilde{\theta} / 2\left(1+\frac{2 v}{\rho^{\prime}}+\rho \cdot \delta^{2}\right) \cdot \delta^{2}, \\
\widehat{V}_{T}= & {\left[\frac{1}{2} \rho+\tan ^{2} \tilde{\theta} / 2\right]-\frac{1}{\rho^{\prime}} \tan ^{2} \tilde{\theta} / 2 } \\
& \times\left(v+\frac{1}{2} \rho \rho^{\prime} \cdot \delta^{2}\right) \cdot \delta^{2}, \\
\widehat{V}_{T^{\prime}}= & {\left[\frac{1}{\rho^{\prime}} \tan ^{2} \tilde{\theta} / 2\right]\left(1-v \rho^{\prime} \cdot \delta^{2}\right) . }
\end{aligned}
$$

Here $m_{v}$ has been assumed to be zero, and the entire lepton mass dependence occurs via the dimensionless parameter

$$
\delta \equiv \frac{m^{\prime}}{\sqrt{\left|Q^{2}\right|}} .
$$

Moreover, in the above we have defined

$$
\begin{aligned}
v & \equiv \frac{\omega}{q}=\frac{\lambda}{\kappa}, \\
\rho & \equiv \frac{\left|Q^{2}\right|}{q^{2}}=\frac{\tau}{\kappa^{2}}=1-v^{2}, \\
\rho^{\prime} & \equiv \frac{q}{\epsilon+\epsilon^{\prime}},
\end{aligned}
$$

which turn out to be related as

$$
\rho^{\prime}=\frac{\tan \tilde{\theta} / 2}{\sqrt{\rho+\tan ^{2} \tilde{\theta} / 2}}
$$

and lie between zero and unity. In passing we note that, using the generalized scattering angle in Eq. (47),

$$
\begin{aligned}
\left|Q^{2}\right| & =4 \epsilon \epsilon^{\prime} \sin ^{2} \tilde{\theta} / 2, \\
v_{0} & =4 \epsilon \epsilon^{\prime} \cos ^{2} \tilde{\theta} / 2 .
\end{aligned}
$$

Finally, for use later we define

$$
\widehat{V}_{L} \equiv \widehat{V}_{C C}-2 v \widehat{V}_{C L}+v^{2} \widehat{V}_{L L}
$$

For comparisons with finite-mass corrections to electron scattering, see [42].

In the extreme relativistic limit (ERL), namely $m^{\prime} \rightarrow 0$, the kinematic factors are obtained by observing that in this situation $\widetilde{\theta}$ becomes $\theta$ and all of the terms containing $\delta$ can be dropped. One then gets

$$
\begin{gathered}
\widehat{V}_{C C} \rightarrow v_{C C}=1, \\
\widehat{V}_{C L} \rightarrow v_{C L}=v, \\
\widehat{V}_{L L} \rightarrow v_{L L}=v^{2}, \\
\widehat{V}_{L} \rightarrow v_{L}=\rho^{2}, \\
\widehat{V}_{T} \rightarrow v_{T}=\frac{1}{2} \rho+\tan ^{2} \theta / 2, \\
\widehat{V}_{T^{\prime}} \rightarrow v_{T^{\prime}}=\tan \theta / 2 \sqrt{\rho+\tan ^{2} \theta / 2,}
\end{gathered}
$$

where the last three kinematic factors coincide with those employed in electron scattering (spin observables, coincidence electron scattering, parity-violating electron scattering, etc.).

In the case of the $\mu=0(C)$ and $3(L)$ components of the vector current, which is assumed to be conserved, it is possible to collapse the contributions down to a single term.
In particular, one has

$$
\begin{aligned}
& R_{C L}^{V V}=-v R_{C C}^{V V} \\
& R_{L L}^{V V}=v^{2} R_{C C}^{V V}
\end{aligned}
$$

Since everything of the purely polar-vector type can be related to a single response, traditionally we call this the longitudinal contribution, defined by the equation $R_{L}^{V V} \equiv R_{C C}^{V V}$. Expressing the sum of the $(\mu, v)=(0,0),(0,3),(3,0)$, and $(3,3)$ contributions, one then ends up with the single term

$$
\widehat{V}_{C C} R_{C C}^{V V}+2 \widehat{V}_{C L} R_{C L}^{V V}+\widehat{V}_{L L} R_{L L}^{V V}=\widehat{V}_{L} R_{L}^{V V} \equiv X_{L}^{V V} .
$$

This collapse into a single expression does not occur for the $A A$ terms. Indeed there one has

$$
\widehat{V}_{C C} R_{C C}^{A A}+2 \widehat{V}_{C L} R_{C L}^{A A}+\widehat{V}_{L L} R_{L L}^{A A} \equiv X_{C / L}^{A A} .
$$

To complete the analysis one should add the two contributions $(\mu, v)=(1,1)$ and $(2,2)$, which yield

$$
\widehat{V}_{T}\left[R_{T}^{V V}+R_{T}^{A A}\right] \equiv X_{T},
$$

and, as well, consider the $V / A$ interference term where $(\mu, v)=(1,2)$ and $(2,1)$,

$$
2 \widehat{V}_{T^{\prime}} R_{T^{\prime}}^{V A} \equiv X_{T^{\prime}}
$$

The full response will then be [see Eq. (52)]

$$
\mathcal{F}_{\chi}^{2}=X_{L}^{V V}+X_{C / L}^{A A}+X_{T}+\chi X_{T^{\prime}}
$$

\section{A. Single-nucleon responses in the $\mathrm{QE}$ region}

The single-nucleon responses in the $\mathrm{QE}$ region all begin with the basic vector and axial-vector currents involving $N \rightarrow N$ matrix elements:

$$
\begin{aligned}
& j_{V}^{\mu}=\bar{u}\left(P^{\prime}\right)\left[F_{1} \gamma^{\mu}+\frac{i}{2 m_{N}} F_{2} \sigma^{\mu \nu} Q_{v}\right] u(P), \\
& j_{A}^{\mu}=\bar{u}\left(P^{\prime}\right)\left[G_{A} \gamma^{\mu}+\frac{1}{2 m_{N}} G_{P} Q^{\mu}\right] \gamma^{5} u(P),
\end{aligned}
$$

with $Q^{\mu}=P^{\prime \mu}-P^{\mu}$. Indeed, in the scaling analyses discussed in Sec. III A, the former was used together with the usual relationship between the Dirac/Pauli form factors and the Sachs form factors, $G_{E}=F_{1}-\tau F_{2}$ and $G_{M}=F_{1}+F_{2}$, to obtain expressions such as those in Eqs. (16) and (17). The total current is then $j^{\mu}=j_{V}^{\mu}-j_{A}^{\mu}$.

In fact, for the purely polar-vector contributions we have

$$
\begin{aligned}
& R_{L}^{V V}=\frac{1}{\rho}\left[G_{E}^{(1)}\right]^{2} \\
& R_{T}^{V V}=2 \tau\left[G_{M}^{(1)}\right]^{2},
\end{aligned}
$$

which were used above. Here $G_{E, M}^{(1)}$ are the nucleon's EM isovector form factors. We reemphasize that, as written, these results contain effects from the motion of the nucleons in the nucleus up to first-order in $\eta_{F}$ and only terms of order $\eta_{F}^{2}$ and beyond have been neglected. 
For the axial-vector current proceeding from Eq. (85) one has

$$
\begin{aligned}
R_{C C}^{A A} & =\frac{v^{2}}{\rho}\left[G_{A}^{\prime(1)}\right]^{2}, \\
R_{C L}^{A A} & =-\frac{v}{\rho}\left[G_{A}^{\prime(1)}\right]^{2}, \\
R_{L L}^{A A} & =\frac{1}{\rho}\left[G_{A}^{\prime(1)}\right]^{2}, \\
R_{T}^{A A} & =2(1+\tau)\left[G_{A}^{(1)}\right]^{2},
\end{aligned}
$$

defining the following combination of axial-vector and induced pseudoscalar form factors

$$
G_{A}^{\prime(1)}(\tau)=G_{A}^{(1)}(\tau)-\tau G_{P}^{(1)}(\tau),
$$

namely, the axial-vector analog of the relationship between the Dirac, Pauli, and Sachs form factors. It is of importance to realize that in the ERL [see Eqs. (71)-(73)] $X_{C / L}^{A A} \rightarrow 0$. Hence this term crucially depends upon the final lepton mass, yielding for the $C / L$ single-nucleon $\mathrm{QE}$ response

$$
X_{C / L}^{A A}=\tan ^{2} \tilde{\theta} / 2\left[G_{A}^{\prime(1)}\right]^{2} \cdot\left(1+\delta^{2}\right) \delta^{2} .
$$

Finally the $V / A$ interference term is

$$
R_{T^{\prime}}^{V A}=2 \sqrt{\tau(1+\tau)} G_{M}^{(1)} G_{A}^{(1)} .
$$

\section{B. Single-nucleon responses in the $\Delta$ region}

In this subsection we consider the elementary reactions

$$
\begin{aligned}
& v_{\mu} p \rightarrow \mu^{-} \Delta^{++}, \\
& v_{\mu} n \rightarrow \mu^{-} \Delta^{+}, \\
& \bar{v}_{\mu} p \rightarrow \mu^{+} \Delta^{0}, \\
& \bar{v}_{\mu} n \rightarrow \mu^{+} \Delta^{-} .
\end{aligned}
$$

The associated currents are [22]

$$
J^{\mu}(q)=\mathcal{T} \bar{u}_{\alpha}^{(\Delta)}\left(p^{\prime}, s^{\prime}\right) \Gamma^{\alpha \mu} u(p, s),
$$

where the isospin factor $\mathcal{T}$ is $\sqrt{3}$ for $\Delta^{++}$and $\Delta^{-}$production and 1 for $\Delta^{+}$and $\Delta^{0}$ production, $u_{\alpha}^{(\Delta)}\left(p^{\prime}, s^{\prime}\right)$ and $u(p, s)$ are the Rarita-Schwinger and Dirac spinors for a $\Delta$ and a nucleon with momenta $p^{\prime}=p+q$ and $p$ and $\operatorname{spin} s^{\prime}$ and $s$, respectively. For the vertex tensor we take [22]

$$
\begin{aligned}
\Gamma^{\alpha \mu}= & {\left[\frac{C_{3}^{V}}{m_{N}}\left(g^{\alpha \mu} q-q^{\alpha} \gamma^{\mu}\right)+\frac{C_{4}^{V}}{m_{N}^{2}}\left(g^{\alpha \mu} q \cdot p^{\prime}-q^{\alpha} p^{\prime \mu}\right)\right.} \\
& \left.+\frac{C_{5}^{V}}{m_{N}^{2}}\left(g^{\alpha \mu} q \cdot p-q^{\alpha} p^{\mu}\right)\right] \gamma_{5}+\left[\frac{C_{3}^{A}}{m_{N}}\left(g^{\alpha \mu} q-q^{\alpha} \gamma^{\mu}\right)\right. \\
& \left.+\frac{C_{4}^{A}}{m_{N}^{2}}\left(g^{\alpha \mu} q \cdot p^{\prime}-q^{\alpha} p^{\prime \mu}\right)+C_{5}^{A} g^{\alpha \mu}+\frac{C_{6}^{A}}{m_{N}^{2}} q^{\alpha} q^{\mu}\right] .
\end{aligned}
$$

We recall that CVC implies $C_{6}^{V}=0$ and PCAC yields $C_{6}^{A}=$ $C_{5}^{A}\left(\mu_{\pi}^{2}+4 \tau\right)^{-1}$, with $\mu_{\pi}=m_{\pi} / m_{N}, m_{\pi}$ being the pion mass.

The hadronic tensor

$$
w^{\mu \nu}=\mu_{\Delta} \operatorname{Tr}\left\{J^{\mu \dagger}(q) J^{v}(q)\right\}
$$

with $\mu_{\Delta}=m_{\Delta} / m_{N}$ as above, can be rewritten in the form

$$
w^{\mu \nu}=\mathcal{T}^{2} \mu_{\Delta} \operatorname{Tr}\left\{P_{\beta \alpha}\left(p^{\prime}\right)\left(\gamma_{0} \Gamma^{\dagger \alpha \mu} \gamma_{0}\right) \Lambda(p) \Gamma^{\beta \nu}\right\},
$$

where

$$
\begin{aligned}
P_{\beta \alpha}\left(p^{\prime}\right)= & \sum_{s^{\prime}} u_{\beta}^{(\Delta)}\left(p^{\prime}, s^{\prime}\right) \bar{u}_{\alpha}^{(\Delta)}\left(p^{\prime}, s^{\prime}\right) \\
= & -\frac{\not p^{\prime}+m_{\Delta}}{2 m_{\Delta}} \\
& \times\left(g_{\beta \alpha}-\frac{2}{3} \frac{p_{\beta}^{\prime} p_{\alpha}^{\prime}}{m_{\Delta}^{2}}+\frac{1}{3} \frac{p_{\beta}^{\prime} \gamma_{\alpha}-p_{\alpha}^{\prime} \gamma_{\beta}}{m_{\Delta}}-\frac{1}{3} \gamma_{\beta} \gamma_{\alpha}\right)
\end{aligned}
$$

is the Rarita-Schwinger projector, while as usual

$$
\Lambda(p)=\frac{\not p+m_{N}}{2 m_{N}}
$$

is the nucleon projector.

A lengthy calculation then yields

$$
w^{\mu v}=w_{V V}^{\mu \nu}+w_{A A}^{\mu \nu}+w_{V A}^{\mu v},
$$

where

$$
\begin{aligned}
w_{V V}^{\mu \nu}= & -w_{1 V}\left(g^{\mu \nu}+\frac{\kappa^{\mu} \kappa^{\nu}}{\tau}\right) \\
& +w_{2 V}\left(\eta^{\mu}+\rho_{\Delta} \kappa^{\mu}\right)\left(\eta^{\nu}+\rho_{\Delta} \kappa^{\nu}\right), \\
w_{A A}^{\mu \nu}= & -w_{1 A}\left(g^{\mu \nu}+\frac{\kappa^{\mu} \kappa^{\nu}}{\tau}\right)+w_{2 A}\left(\eta^{\mu}+\rho_{\Delta} \kappa^{\mu}\right)\left(\eta^{\nu}+\rho_{\Delta} \kappa^{\nu}\right) \\
& -u_{1 A} \frac{\kappa^{\mu} \kappa^{\nu}}{\tau}+u_{2 A}\left(\kappa^{\mu} \eta^{\nu}+\eta^{\mu} \kappa^{\nu}\right),
\end{aligned}
$$

and

$$
w_{V A}^{\mu \nu}=2 i w_{3} \epsilon^{\alpha \beta \mu \nu} \eta_{\alpha} \kappa_{\beta}
$$

are the vector-vector, axial-axial, and vector-axial interference hadronic tensors, respectively. The functions $u_{1 \mathrm{~A}}$ and $u_{2 \mathrm{~A}}$ reflect the nonconservation of the axial-vector current and will be discussed in the Appendix. The functions $w_{i}$ entering above are obtained by performing the traces in Eq. (101) and using the on-shell conditions $\eta^{2}=1$ and $\eta \cdot \kappa=\tau \rho_{\Delta}=$ $\tau+\frac{1}{4}\left(\mu_{\Delta}^{2}-1\right)$. The results are collected in the Appendix.

\section{Nuclear cross sections and response functions}

To compute the cross section in Eq. (45), the factor $\mathcal{F}_{\chi}^{2}$ in Eq. (83) is required, and thus the nuclear response functions $R_{i}$ are needed. The last can be expressed in terms of the nuclear tensor $W^{\mu \nu}$ according to

$$
\begin{aligned}
& R_{L}^{V V}=W_{V V}^{00}, \\
& R_{C C}^{A A}=W_{A A}^{00}, \\
& R_{C L}^{A A}=-W_{A A}^{03}, \\
& R_{L L}^{A A}=W_{A A}^{33}, \\
& R_{T}^{V V}=W_{V V}^{11}+W_{V V}^{22}, \\
& R_{T}^{A A}=W_{A A}^{11}+W_{A A}^{22}, \\
& R_{T^{\prime}}^{V A}=-i W_{V A}^{12} .
\end{aligned}
$$


Since in the QE domain the nuclear tensor is well known, we focus here on the $\Delta$ sector, where using Eq. (15) $W^{\mu v}$ reads [38]

$$
\begin{aligned}
{\left[W^{\mu \nu}\right]^{\Delta} } & =\frac{3 \mathcal{N}}{8 m_{N} \eta_{F}^{3} \kappa} \int_{\epsilon_{0}}^{\epsilon_{F}} w^{\mu \nu}(\epsilon) \theta\left(\epsilon_{F}-\epsilon_{0}\right) d \epsilon \\
& =\frac{1}{2} \Lambda_{0} f_{\mathrm{RFG}}\left(\psi_{\Delta}\right) U^{\mu \nu},
\end{aligned}
$$

where $\mathcal{N}=Z$ for reactions on protons, (95) and (97), whereas $\mathcal{N}=N$ for reactions on neutrons, (96) and (98). Again, only isovector form factors enter into the equation.

As discussed in Sec. III B, the RFG superscaling function in the $\Delta$ domain is given by

$$
f_{\mathrm{RFG}}\left(\psi_{\Delta}\right)=\frac{3}{4}\left(1-\psi_{\Delta}^{2}\right) \theta\left(1-\psi_{\Delta}^{2}\right)
$$

and

$$
U^{\mu \nu}=\frac{1}{\epsilon_{F}-\epsilon_{0}} \int_{\epsilon_{0}}^{\epsilon_{F}} w^{\mu \nu}(\epsilon) d \epsilon
$$

where

$$
\epsilon_{0}=\kappa \sqrt{\rho_{\Delta}^{2}+1 / \tau}-\lambda \rho_{\Delta}
$$

represents the minimum energy a nucleon should have in order to take part in the $\Delta$ electro-excitation process and $\epsilon_{F}=\sqrt{1+\eta_{F}^{2}}$ is the Fermi energy. Note that from Eq. (26), one has

$$
\psi_{\Delta}=\left[\frac{1}{\xi_{F}}\left(\epsilon_{0}-1\right)\right]^{1 / 2} \times\left\{\begin{array}{l}
+1 \lambda \geqslant \lambda_{\Delta}^{0}, \\
-1 \lambda \leqslant \lambda_{\Delta}^{0},
\end{array}\right.
$$

with $\lambda_{\Delta}^{0}=\frac{1}{2}\left[\sqrt{\mu_{\Delta}^{2}+4 \kappa^{2}}-1\right]$ using Eq. (27).

The required components of the $U^{\mu v}$ tensor can be explicitly computed using Eqs. (107)-(109) and performing the integral in Eq. (117). They turn out to be

$$
U_{V V}^{00}=\frac{\kappa^{2}}{\tau}\left[-w_{1 V}(\tau)+\left(1+\tau \rho_{\Delta}^{2}\right) w_{2 V}(\tau)+\mathcal{D}(\kappa, \tau) w_{2 V}(\tau)\right],
$$

$$
\begin{aligned}
U_{A A}^{00}=\frac{\kappa^{2}}{\tau}[ & \left.-w_{1 A}(\tau)+\left(1+\tau \rho_{\Delta}^{2}\right) w_{2 A}(\tau)+\mathcal{D}(\kappa, \tau) w_{2 A}(\tau)\right] \\
-\frac{\lambda^{2}}{\tau} & u_{1 A}(\tau)+\lambda\left(\epsilon_{F}+\epsilon_{0}\right) u_{2 A}(\tau), \\
U_{A A}^{03}= & \left(\frac{\lambda}{\kappa}\right)\left\{\frac { \kappa ^ { 2 } } { \tau } \left[-w_{1 A}(\tau)+\left(1+\tau \rho_{\Delta}^{2}\right) w_{2 A}(\tau)\right.\right. \\
& \left.+\mathcal{D}(\kappa, \tau) w_{2 A}(\tau)\right]-\frac{\kappa^{2}}{\tau} u_{1 A}(\tau) \\
& \left.+\left[\frac{\lambda^{2}+\kappa^{2}}{2 \lambda}\left(\epsilon_{F}+\epsilon_{0}\right)-\tau \rho_{\Delta}\right] u_{2 A}(\tau)\right\} \\
U_{A A}^{33}= & \left(\frac{\lambda}{\kappa}\right)^{2}\left\{\frac { \kappa ^ { 2 } } { \tau } \left[-w_{1 A}(\tau)+\left(1+\tau \rho_{\Delta}^{2}\right) w_{2 A}(\tau)\right.\right. \\
& \left.+\mathcal{D}(\kappa, \tau) w_{2 A}(\tau)\right]-\frac{\kappa^{4}}{\tau \lambda^{2}} u_{1 A}(\tau) \\
& \left.+\frac{\kappa^{2}}{\lambda^{2}}\left[\lambda\left(\epsilon_{F}+\epsilon_{0}\right)-2 \tau \rho_{\Delta}\right] u_{2 A}(\tau)\right\},
\end{aligned}
$$

for pieces of the tensor having $\mu$ or $v$ equal to 0 or 3 ; while for transverse projections one has

$$
\begin{array}{r}
U_{V V}^{11}+U_{V V}^{22}=2 w_{1 V}(\tau)+\mathcal{D}(\kappa, \tau) w_{2 V}(\tau), \\
U_{A A}^{11}+U_{A A}^{22}=2 w_{1 A}(\tau)+\mathcal{D}(\kappa, \tau) w_{2 A}(\tau), \\
U_{V A}^{12}=2 i \sqrt{\tau\left(1+\tau \rho_{\Delta}^{2}\right)}\left[1+\mathcal{D}^{\prime}(\kappa, \tau)\right] w_{3}(\tau),
\end{array}
$$

where

$$
\begin{aligned}
\mathcal{D}(\kappa, \tau)= & \frac{\tau}{\kappa^{2}}\left[\frac{1}{3}\left(\epsilon_{F}^{2}+\epsilon_{0} \epsilon_{F}+\epsilon_{0}^{2}\right)\right. \\
& \left.+\lambda \rho_{\Delta}\left(\epsilon_{F}+\epsilon_{0}\right)+\lambda^{2} \rho_{\Delta}^{2}\right]-1-\tau \rho_{\Delta}^{2}, \\
\mathcal{D}^{\prime}(\kappa, \tau)= & \frac{1}{\kappa} \sqrt{\frac{\tau}{1+\tau \rho_{\Delta}^{2}}}\left[\lambda \rho_{\Delta}+\frac{1}{2}\left(\epsilon_{F}+\epsilon_{0}\right)\right]-1 .
\end{aligned}
$$

Thus we are now in a position to assemble the various factors and provide predictions for the charge-changing neutrino reaction cross sections. The single-nucleon cross sections in both the $\mathrm{QE}$ and $\Delta$ regions are given above and, from the scaling analysis presented in Sec. III, we experimentally determine scaling functions $f^{\mathrm{QE}}\left(\psi_{\mathrm{QE}}^{\prime}\right)$ and $f^{\Delta}\left(\psi_{\Delta}^{\prime}\right)$. As usual, we use scaling variables $\psi_{\mathrm{QE}}^{\prime}$ and $\psi_{\Delta}^{\prime}$ in which we include a small energy shift by replacing $\omega$ with $\omega-E_{\text {shift }} \equiv \omega^{\prime}, \lambda \rightarrow \lambda^{\prime}$, etc. For charge-changing neutrino reactions one proceeds to analogous states in the neighboring nuclei, which are shifted from their positions in the target nucleus at least by the Coulomb energy. Accordingly, while having a very small effect, in this work we modify $E_{\text {shift }}$ from its value as obtained in studies of electron scattering by these additional amounts. In the case of mass-12 this means that upon comparing the excitation energy of the analog of the nitrogen and boron ground states (i.e., the energy $15.110 \mathrm{MeV}$ of the lowest $J^{\pi} T=1^{+} 1$ state in carbon), we should add $16.827-15.110=1.717 \mathrm{MeV}$ when making transitions to nitrogen (neutrino reactions) and subtract $15.110-13.880=1.230 \mathrm{MeV}$ when making transitions to boron (antineutrino reactions) from the canonical value of $E_{\text {shift }}=20 \mathrm{MeV}$. These are then used in the definitions of $\psi^{\prime}$ in each case. Note that we use the correct ground state masses of the three nuclei in establishing the lepton scattering kinematics discussed in Sec. II.

Finally, in computing the results to be presented in the next section the following form factors are employed. For the vector sector we use the Höhler parametrization 8.2 [36] and for the axial-vector sector we use

$$
\begin{aligned}
G_{D}^{A}(\tau) & =\left(1+\lambda_{D}^{A} \tau\right)^{-2}, \\
G_{A}^{(1)}(\tau) & =g_{A} G_{D}^{A}(\tau), \\
G_{P}^{(1)}(\tau) & =\frac{1}{1 / \lambda_{A}^{\prime}+\tau} G_{A}^{(1)}(\tau),
\end{aligned}
$$

with $\lambda_{D}^{A}=3.32$ (corresponding to axial-vector mass $M_{A}=$ $1032 \mathrm{MeV}), \lambda_{A}^{\prime}=\left(2 m_{N} / m_{\pi}\right)^{2}=180$, and $g_{A}=1.26$. 

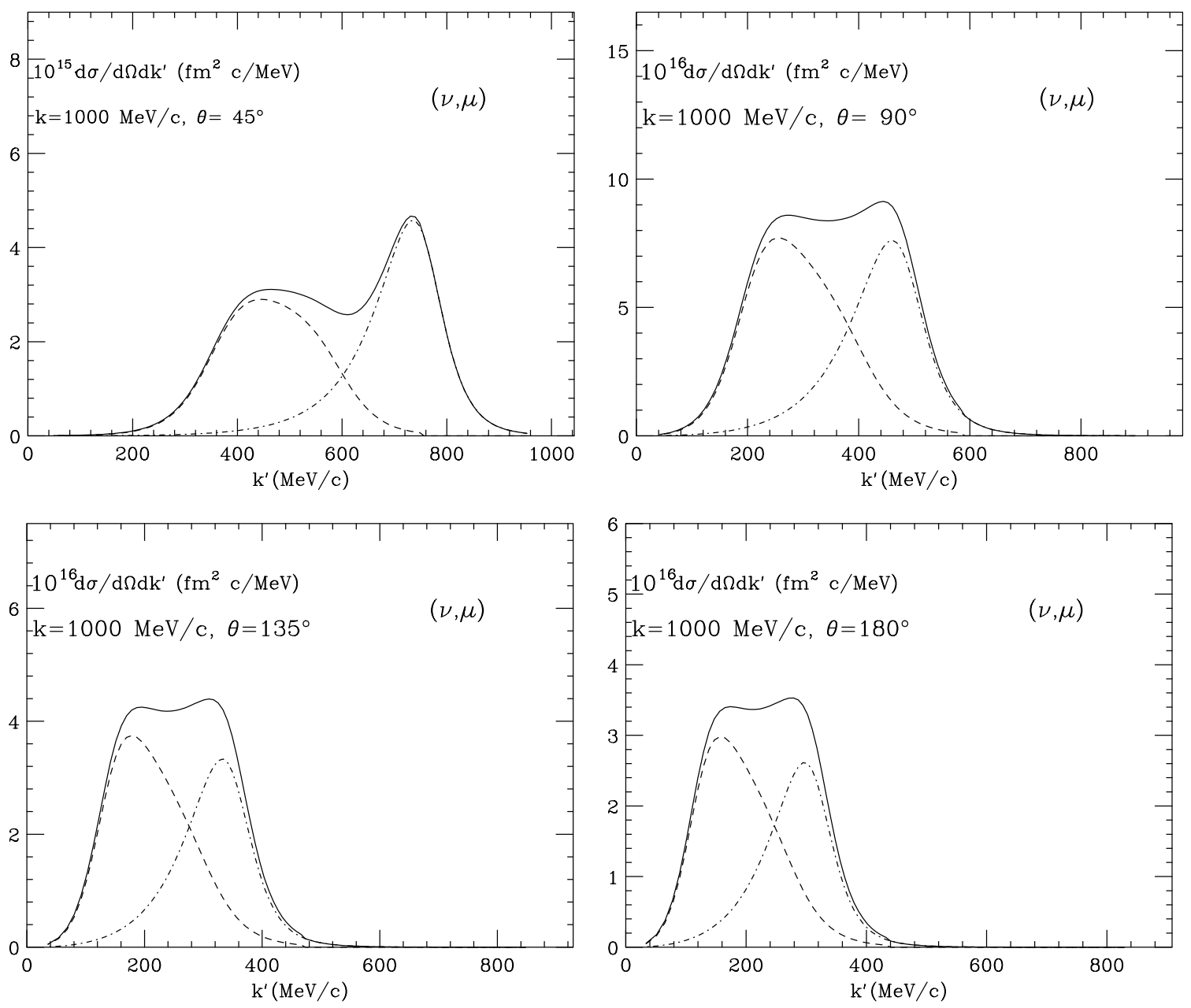

FIG. 6. Charge-changing neutrino reactions $\left(v_{\mu}, \mu^{-}\right)$on ${ }^{12} \mathrm{C}$ for $1 \mathrm{GeV}$ neutrinos and neutrino-muon scattering angles of 45 , 90 , 135, and 180 degrees. The cross sections are plotted versus the final-state muon momentum $k^{\prime}$. The dash-dotted curve gives the QE contribution, the dashed curve the $\Delta$ contribution, and the solid curve the total. As discussed in the text, results for values of $k^{\prime}$ lying below the $\Delta$ peak (higher excitation energies than that of the $\Delta$ ) must be viewed with caution.

\section{NEUTRINO CROSS-SECTION PREDICTIONS}

The inclusive charge-changing neutrino reaction cross sections ${ }^{12} \mathrm{C}\left(v_{\mu}, \mu^{-}\right)$and ${ }^{12} \mathrm{C}\left(\bar{v}_{\mu}, \mu^{+}\right)$that result from the scaling analyses presented above are shown in Figs. 6 and 7 , respectively. A neutrino or antineutrino energy of $1 \mathrm{GeV}$ has been selected as being representative of kinematics where the scaling approach should be expected to work well and a selection of scattering angles (between incident neutrino or antineutrino and produced charged muon) has been made. Note that since the predictions shown here are given as functions of the muon momentum $k^{\prime}$, the QE peak lies to the right (i.e., lower excitation energy) of the $\Delta$ peak (higher excitation energy). Note that the Coulomb distortion correction from Eq. (49) has been included here, although for simplicity we labeled the figures using $k^{\prime}$ rather than $k_{\infty}^{\prime}$. As discussed above, the predictions at momenta to the left of the $\Delta$ peak (excitations lying above the $\Delta$ region) are unreliable because our scaling approach does not fully account for meson production, including resonances other than the $\Delta$, and deep inelastic scattering processes.
Corresponding angular distributions are shown in Fig. 8 for kinematics chosen to lie at the peaks of the $\mathrm{QE}$ (solid curves) and $\Delta$ (dashed curves)

One striking feature of the results is the dramatic differences seen in comparing neutrino and antineutrino cross sections at backward angles. The latter are typically about two orders of magnitude smaller than the former under those conditions. This is because the transverse (vector and axial-vector) contribution to both the $\mathrm{QE}$ and $\Delta$ responses is accidentally roughly the same in magnitude as the one arising from the $V / A$ interference for large scattering angles at the chosen energy. Specifically, for instance in the QE case, one has $X_{T}$ roughly equal to $X_{T^{\prime}}$ [see Eqs. (81), (82), (87), (91), and (94)]. For neutrinos, these constructively interfere, whereas for antineutrinos they tend to cancel and produce much reduced cross sections. Indeed, the cancellation is so severe that the $V V(C C, C L$, and $L L)$ and $A A(C C, C L$, and $L L)$ terms can yield significant contributions to the total cross section. In the $\mathrm{QE}$ region these $V V$ terms yield as much as $1 / 3$ of the cross section, while the $A A$ terms are negligible. In contrast, in the $\Delta$ region 

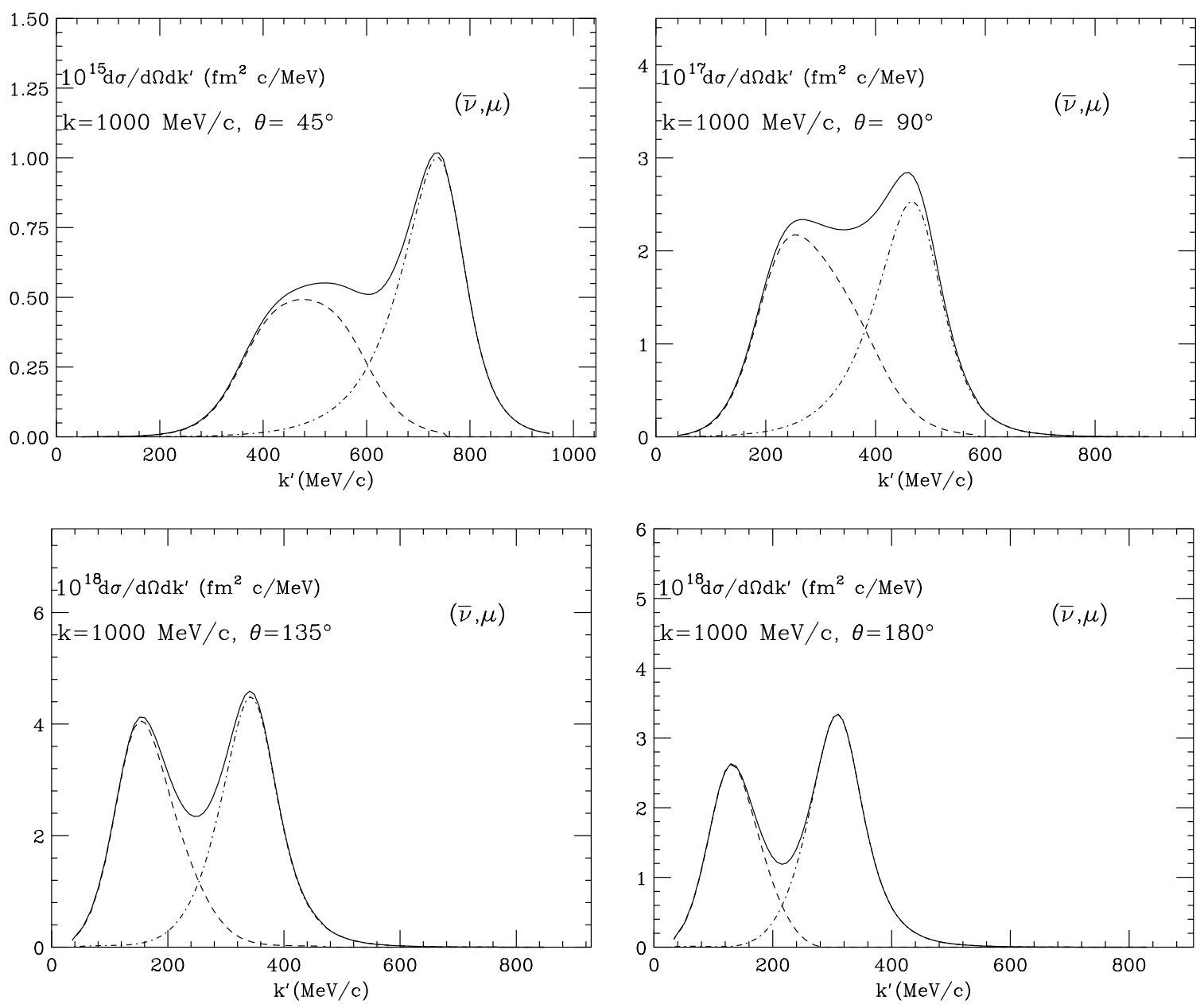

FIG. 7. As for Fig. 6, but now for antineutrino reactions $\left(\bar{v}_{\mu}, \mu^{+}\right)$.

the reverse is true, with the $A A(C C, C L$, and $L L)$ terms even providing the majority of the cross section at large angles in this case. Small changes in the model (for example, the inclusion of MEC) could have very large effects on the predictions for antineutrinos; hence the results shown in this case, especially for large scattering angles, should be viewed with caution. The important observation is that the antineutrino cross sections are predicted to be strongly suppressed due to the accidental cancellation.

For neutrinos, where no such strong cancellation occurs, the cross sections are typically dominated by the $V V(T), A A(T)$, and $V A\left(T^{\prime}\right)$ contributions. In the $\mathrm{QE}$ region the $V V(C C, C L$, and $L L$ ) pieces contribute only about $5 \%$ at 45 degrees and fall to negligible corrections at backward angles, whereas the $A A(C C, C L$, and $L L)$ contributions are negligible for all angles considered. In the $\Delta$ regions the converse is true: the $A A(C C$, $C L$, and $L L$ ) pieces contribute about $5 \%$ or less at 45 degrees and fall to negligible corrections at backward angles, whereas the $V V(C C, C L$, and $L L)$ contributions are negligible for all angles considered.

The fact that the underlying transverse vector and axialvector matrix elements are comparable in magnitude for the kinematics being explored in this work (hence the antineutrino suppression discussed above) has consequences for the uncertainties expected in the predictions made here for neutrinos. We recall from our treatment above that effects from MECs and their associated correlations are not present for these predictions, since they were ignored in analyzing the electron scattering cross sections. Note, however, that the error incurred by this may be less here than for electron scattering: the transverse contributions to the neutrino reaction cross sections involve both polar- and axial-vector matrix elements with both one-body, impulsive contributions (included here) and twobody MEC/correlation contributions (not included). From past studies we know that the latter occur primarily in the transverse channel, but not in the longitudinal channel for electron scattering at the kinematics of interest here. That is, the vector $\mathrm{MEC} /$ correlation effects occur primarily in the transverse channel for $\mathrm{QE}$ and $\Delta$ kinematics at high energies. In contrast, because of the factor $\gamma_{5}$ that enters in axial-vector currents, the converse is true for axial-vector contributions; accordingly, to leading order, one does not expect large corrections of this type for the axial-vector contributions. As a consequence, the residual effect seen in Fig. 2 at large negative $\psi_{\Delta}^{\prime}$ and attributed to MEC effects and their associated correlations, which amounted to roughly $10-15 \%$ of the total cross section 

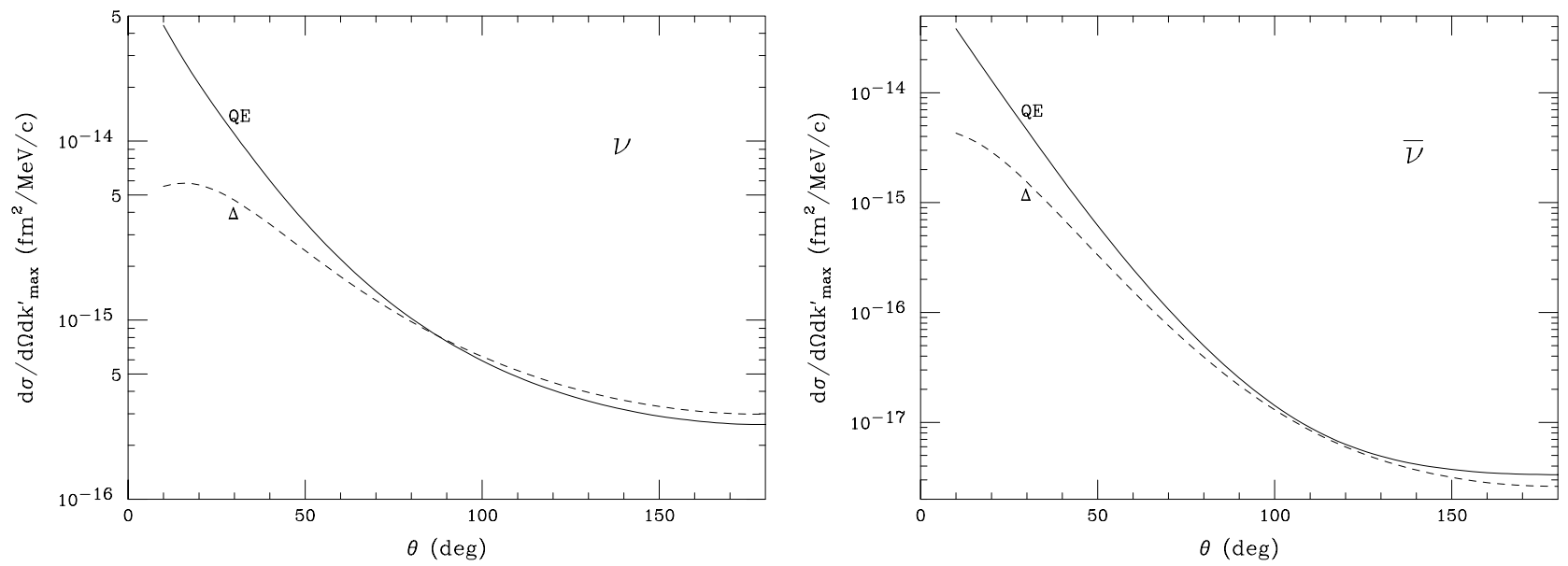

FIG. 8. Angular distributions for the results in Figs. 6 and 7 at the tops of the QE and $\Delta$ peaks. Since the neutrino or antineutrino energy is fixed to $1 \mathrm{GeV}$ and the kinematics are chosen to be at $\psi_{\mathrm{QE}}^{\prime}=0$ and $\psi_{\Delta}^{\prime}=0$, the muon momentum $k^{\prime}$ must vary with $\theta$.

in that region of inelasticity, measures the uncertainty in the vector contributions. Since these are only roughly $1 / 2$ of the total for neutrinos, with the axial-vector transverse contributions accounting for the other half, the overall impact of the neglected MEC/correlation contributions is likewise only half as large, namely, providing less than $10 \%$ uncertainty to the neutrino cross-section predictions made here.

For completeness, we show in Fig. 9 a comparison of a typical neutrino reaction cross section obtained using the full results with $f^{\mathrm{QE}}$ and $f^{\Delta}$ deduced directly from electron scattering data with the cross section obtained using the RFG. As noted earlier, modeling done on the basis of mean-field theory is very similar to the RFG result, since at the relatively high energies of interest the dynamical effects embodied in an effective mass are expected to be small (most recent

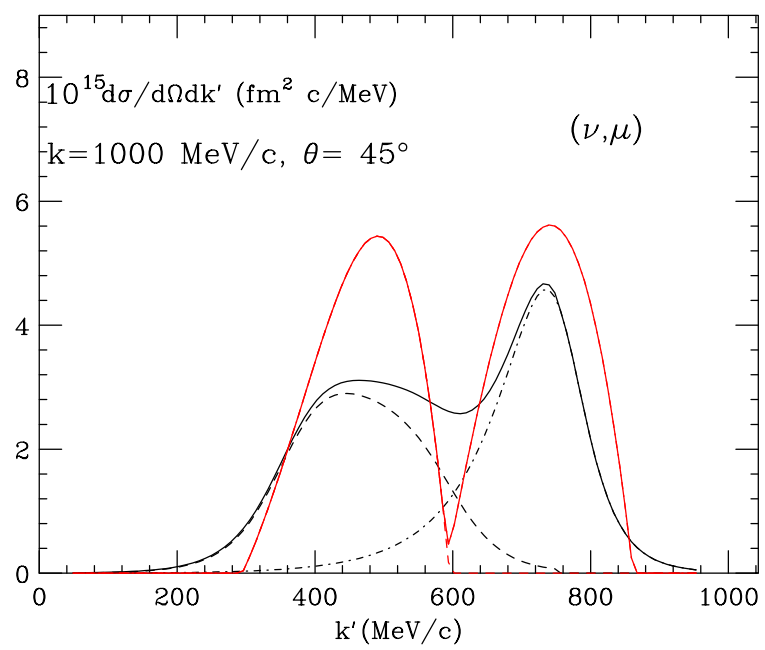

FIG. 9. (Color online) Neutrino reaction cross sections as in Fig. 6 for $\theta=45$ degrees, showing a comparison of the full results obtained using the empirical scaling functions $f^{\mathrm{QE}}$ and $f^{\Delta}$ as discussed in the text with results obtained using the RFG scaling function $f_{\mathrm{RFG}}$ (heavier lines). The former lie somewhat lower and extend over a wider range in $k^{\prime}$ than the latter. relativistic mean-field theory studies predict that $m^{*}$ reverts almost to the nucleon mass for the kinematics of interest). Likewise, relativized shell-model predictions are close to the RFG predictions; moreover, RPA correlations are expected to be relatively small for the high energies involved. Thus, the RFG predictions effectively represent a larger set of models. Therefore, as can be seen in the figure, all differ significantly from the scaling predictions. Given the success of the scaling approach in studies of inclusive electron scattering for the kinematic region under study we expect that neutrino reaction cross sections also obtained using scaling ideas to be more robust than those based directly on existing models.

\section{CONCLUSIONS}

We begin by summarizing the approach followed in the present study.

The first step is to explore the scaling behavior of inclusive electron scattering for relatively high energies (several hundred $\mathrm{MeV}$ to a few $\mathrm{GeV}$ ) in the kinematic region extending from the scaling region that lies below the QE peak, through the QE peak region, and up to the peak where $\Delta$ excitation is the dominant process. Upon examining the longitudinal contribution, one finds superscaling; i.e., it is possible to find a scaling function $f^{\mathrm{QE}}$ which, when plotted versus an appropriate scaling variable $\psi_{\mathrm{QE}}^{\prime}$, is seen to superscale. This means that the results are independent of both the momentum transfer (scaling of the first kind) and the particular nuclear species (scaling of the second kind). The assumption is made that this universal scaling function embodies the basic nuclear dynamics in the problem. Implicit in this, and borne out by modeling, is the observation that apparently MEC effects with their associated correlations and inelastic processes such as $\Delta$ excitation are not large contributors to the longitudinal response.

The second step is to use the universal scaling function $f^{\mathrm{QE}}$ to obtain that part of the transverse response that is due to impulsive processes, namely, those that arise only from elastic $e N$ scattering from nucleons in the nucleus. 
Upon subtracting this contribution from the total transverse response, one finds a residual which is assumed to be due to the MEC/correlation effects and to inelastic $e N$ scattering from nucleons in the nucleus. From modeling we know that such effects are predominantly transverse and hence they naturally occur in this channel but are not very important in the longitudinal channel. Moreover, again from modeling the various processes, we expect that the MEC/correlation effects are relatively small corrections for the kinematics of interest and accordingly attribute most of the residual to impulsive, inelastic $e N$ scattering, especially to contributions which arise from $N \rightarrow \Delta$ transitions. When the residual is analyzed in terms of an appropriate scaling function $f^{\Delta}$ by dividing the residual response by the elementary $N \rightarrow \Delta$ cross section and plotted versus an appropriate scaling variable $\psi_{\Delta}^{\prime}$ which incorporates the kinematics of the inelastic transition, we again find a reasonably successful new kind of scaling, at least for kinematics where the concept is expected to work. Specifically, this success is only found for excitations up to the peak of the region where the $\Delta$ dominates, but not beyond. This is not unexpected, since the approach taken in the present work has been tailored to only work when the $\Delta$ provides the basic driving process. Also, deviations are seen in the region where the $\mathrm{QE}$ and $\Delta$ responses overlap, and there one does expect corrections from MEC effects and their associated correlations, while small, nevertheless to be necessary for a fully successful representation of the total response. A check is made by reassembling the complete inclusive $\left(e, e^{\prime}\right)$ cross section for the kinematic region of interest, typically finding errors of $10 \%$ or less.

Thus, the first goal of this work has been met: we found a very good representation of inclusive electron scattering at relatively high energies for the region of excitation extending up to the peak of the $\Delta$. It should be noted that direct modeling (i.e., relativistic modeling, since nonrelativistic approaches are known to fail badly for the kinematics of interest) yields electron scattering cross sections that are valid only at about the $25 \%$ level or worse, while here our goal has been to use the scaling approach to do better.

The second major objective in this study has been to predict charge-changing neutrino and antineutrino cross sections for the same range of kinematics. Using the scaling approach in reverse, we take the empirically determined scaling functions $f^{\mathrm{QE}}$ and $f^{\Delta}$ together with the appropriate $N \rightarrow N$ and $N \rightarrow \Delta$ charge-changing weak interaction cross sections to obtain the inclusive $v A$ and $\bar{v} A$ cross sections for the case of ${ }^{12} \mathrm{C}$. Given the above statements of where the approach taken in the present work should be valid, we believe that these predictions should be the best currently available for few-GeV neutrino reactions in the kinematic region that includes the full $\mathrm{QE}$ response and the $\Delta$ response up to its maximum. From our analyses of MEC contributions and how they enter in the relevant vector and axial-vector responses, we expect that corrections from such processes account for only about $10 \%$ of the total cross section. Note that while our focus has been on the case of carbon, we know from our previous studies of second-kind scaling that it is straightforward to produce predictions for other nuclei as well. In particular, while only selected predictions are given in the present work, further results at different kinematics and for other nuclei may be obtained by contacting our collaboration.

Finally, we mention our intentions for future work. The most straightforward project will be to extend the scaling approach to obtain predictions for neutral current neutrino and antineutrino scattering from nuclei for similar kinematics; studies of this type are currently in progress. Also, given recent work on inelastic $e N$ processes and their role in $e A$ inclusive cross sections undertaken by the same collaboration, our intent is to explore a more microscopic model for all of these reactions. While this project is relatively straightforward as well, what is lacking before it can be realized is a completion of our ongoing studies of MEC effects, together with the correlations they require because of gauge invariance; studies of this type are also in progress. Finally, there is the issue of explaining the specific nature of the scaling functions themselves. While there are indications that contributions from high Fourier components in the nuclear ground state are probably responsible for the detailed nature of these functions, a fully satisfactory relativistic treatment of them is presently lacking. Until one becomes available it appears that the best approach is to take the scaling functions directly from experiment, as we have done in the present work.

\section{ACKNOWLEDGMENTS}

This work was partially supported by DGI (Spain), FEDER (under Contract Nos. BFM2002-03218, BFM2002-03315, and FPA2002-04181-C04-04), the Junta de Andalucía, and the INFN-CICYT collaboration agreement (project "Study of Relativistic Dynamics in Electron and Neutrino Scattering"). It was also supported in part (T.W.D.) by the U.S. Department of Energy under cooperative research agreement No. DEFC02-94ER40818 and in part (I.S.) by the Schweizerische Nationalfonds. The authors wish to thank members of the various neutrino oscillation collaborations for very helpful discussions of ongoing neutrino oscillation experiments.

\section{APPENDIX}

Performing the traces in Eq. (101) one get

$$
\begin{aligned}
w_{1 V}= & \left(\frac{\mathcal{T}^{2}}{3}\right) \frac{1}{4 \mu_{\Delta}^{2}}\left[4 \tau+\left(\mu_{\Delta}-1\right)^{2}\right]\left\{\left[16 \tau^{2}+8 \tau\left(\mu_{\Delta}+1\right)\right.\right. \\
+ & \left.\left(\mu_{\Delta}+1\right)^{2}\left(3 \mu_{\Delta}^{2}+1\right)\right] C_{3}^{V 2}+\mu_{\Delta}^{2}\left[\left(1+4 \tau-\mu_{\Delta}^{2}\right) C_{4}^{V}\right. \\
+ & \left.\left(1-4 \tau-\mu_{\Delta}^{2}\right) C_{5}^{V}\right]^{2}+\mu_{\Delta} C_{3}^{V}\left(1+4 \tau-2 \mu_{\Delta}-3 \mu_{\Delta}^{2}\right) \\
\times & {\left.\left[\left(1+4 \tau-\mu_{\Delta}^{2}\right) C_{4}^{V}+\left(1-4 \tau-\mu_{\Delta}^{2}\right) C_{5}^{V}\right]\right\}, \quad(\mathrm{A} 1) } \\
w_{2 V}= & \left(\frac{\mathcal{T}^{2}}{3}\right) \frac{4 \tau}{\mu_{\Delta}^{2}}\left\{\left(1+4 \tau+3 \mu_{\Delta}^{2}\right) C_{3}^{V 2}\right. \\
& +\left[4 \tau+\left(\mu_{\Delta}-1\right)^{2}\right]\left[\mu_{\Delta}^{2}\left(C_{4}^{V}+C_{5}^{V}\right)^{2}+4 \tau C_{5}^{V 2}\right] \\
& +\mu_{\Delta}\left[\left(1+4 \tau-4 \mu_{\Delta}+3 \mu_{\Delta}^{2}\right)\right. \\
& \left.\left.\times\left(C_{4}^{V}+C_{5}^{V}\right)+8 \tau C_{5}^{V}\right] C_{3}^{V}\right\},
\end{aligned}
$$


for the vector contributions, whereas they yield

$$
\begin{aligned}
w_{1 A}= & \left(\frac{\mathcal{T}^{2}}{3}\right) \frac{1}{4 \mu_{\Delta}^{2}}\left[4 \tau+\left(\mu_{\Delta}+1\right)^{2}\right]\left\{\left[16 \tau^{2}-8 \tau\left(\mu_{\Delta}-1\right)\right.\right. \\
& \left.+\left(\mu_{\Delta}-1\right)^{2}\left(3 \mu_{\Delta}^{2}+1\right)\right] C_{3}^{A 2} \\
& +\mu_{\Delta}^{2}\left[\left(1+4 \tau-\mu_{\Delta}^{2}\right) C_{4}^{A}-2 C_{5}^{V}\right]^{2} \\
& +\mu_{\Delta} C_{3}^{A}\left(1+4 \tau+2 \mu_{\Delta}-3 \mu_{\Delta}^{2}\right) \\
& \left.\times\left[\left(1+4 \tau-\mu_{\Delta}^{2}\right) C_{4}^{A}-2 C_{5}^{A}\right]\right\} \\
w_{2 A}= & \left.\frac{\mathcal{T}^{2}}{3}\right) \frac{4 \tau}{\mu_{\Delta}^{2}}\left\{\left(1+4 \tau+3 \mu_{\Delta}^{2}\right) C_{3}^{A 2}\right. \\
& +\left[4 \tau+\left(\mu_{\Delta}+1\right)^{2}\right]\left(\mu_{\Delta}^{2} C_{4}^{A 2}+\frac{C_{5}^{A 2}}{4 \tau}\right) \\
& \left.+\mu_{\Delta}\left[\left(1+4 \tau+4 \mu_{\Delta}+3 \mu_{\Delta}^{2}\right) C_{4}^{A}+2 C_{5}^{A}\right] C_{3}^{A}\right\} \\
\left.u_{2 A}\right\} & \left(\frac{\mathcal{T}^{2}}{3}\right) \frac{1}{4 \tau \mu_{\Delta}^{2}}\left[4 \tau+\left(\mu_{\Delta}+1\right)^{2}\right]\left(C_{5}^{A}-4 \tau C_{6}^{A}\right) \\
& \times\left[8 \tau \mu_{\Delta}\left(C_{3}^{A}+\mu_{\Delta} C_{4}^{A}\right)+C_{5}^{A}\left(4 \tau-\mu_{\Delta}^{2}+1\right)\right] \\
& +\left\{C_{5}^{A}\left[48 \tau^{2}-\left(\mu_{\Delta}^{2}-1\right)^{2}+8 \tau\left(\mu_{\Delta}^{2}+1\right)\right]\right. \\
& +4 \tau\left[4 \mu_{\Delta}\left(C_{3}^{A}+\mu_{\Delta} C_{4}^{A}\right)\left(4 \tau+\mu_{\Delta}^{2}-1\right)\right. \\
u_{1 A}= & \left.\left(\frac{\mathcal{T}^{2}}{3}\right) \frac{1}{16 \tau \mu_{\Delta}^{2}}\left[4 \tau+\left(\mu_{\Delta}^{2}+8 \tau\left(\mu_{\Delta}^{2}+1\right)\right]\right]\right\}
\end{aligned}
$$

for the axial-vector contributions and

$$
\begin{aligned}
w_{3}= & \left(\frac{\mathcal{T}^{2}}{3}\right) \frac{1}{2 \mu_{\Delta}^{2}}\left\{\mu_{\Delta}\left[2 C_{5}^{A}-\left(4 \tau-\mu_{\Delta}^{2}+1\right) C_{4}^{A}\right]\right. \\
& \times\left[\left(1+4 \tau+4 \mu_{\Delta}+3 \mu_{\Delta}^{2}\right) C_{3}^{V}-\mu_{\Delta}\right. \\
& \left.\times\left[\left(4 \tau-\mu_{\Delta}^{2}+1\right) C_{4}^{V}-\left(4 \tau+\mu_{\Delta}^{2}-1\right) C_{5}^{V}\right]\right] \\
& -C_{3}^{A}\left[2\left(1+8 \tau+16 \tau^{2}+2 \mu_{\Delta}^{2}-3 \mu_{\Delta}^{4}\right) C_{3}^{V}\right. \\
& +\mu_{\Delta}\left(1+4 \tau-4 \mu_{\Delta}+3 \mu_{\Delta}^{2}\right)\left[\left(4 \tau-\mu_{\Delta}^{2}+1\right)\right. \\
& \left.\left.\left.\times C_{4}^{V}-\left(4 \tau+\mu_{\Delta}^{2}-1\right) C_{5}^{V}\right]\right]\right\},
\end{aligned}
$$

for the interference pieces. Since the axial-vector current is not conserved, $w_{1}$ and $w_{2}$ are not sufficient to set up the $A A$ hadronic tensor: hence, two extra functions $u_{1 A}$ and $u_{2 A}$, which vanish if $C_{5}^{A}=4 \tau C_{6}^{A}$, come out from the traces.

For the empirical functions entering above we take [22]

$$
\begin{gathered}
C_{3}^{V}(\tau)=\frac{2.05}{\left(1+\left|Q^{2}\right| / 0.54 G e V^{2}\right)^{2}}, \\
C_{4}^{V}(\tau)=-\frac{C_{3}^{V}}{\mu_{\Delta}},
\end{gathered}
$$

$$
\begin{gathered}
C_{5}^{V}(\tau)=0, \\
C_{3}^{A}(\tau)=0, \\
C_{4}^{A}(\tau)=- \\
\times\left(1+\frac{\left|Q^{2}\right|}{(1.28)^{2} G e V^{2}}\right)^{-2}, \\
C_{5}^{A}(\tau)=1.2\left(1-\frac{1.21\left|Q^{2}\right|}{2 G e V^{2}+\left|Q^{2}\right|}\right) \\
\times\left(1+\frac{\left|Q^{2}\right|}{(1.28)^{2} G e V^{2}}\right)^{-2}, \\
C_{6}^{A}(\tau)=C_{5}^{A}(\tau) \frac{m_{N}^{2}}{m_{\pi}^{2}+\left|Q^{2}\right|}=\frac{C_{5}^{A}}{4 \tau+\mu_{\pi}^{2}},
\end{gathered}
$$

which, inserted into Eqs. (A2)-(A7), lead to

$$
\begin{gathered}
w_{1 V}=\left(\frac{\mathcal{T}^{2}}{3}\right) \frac{1}{4 \mu_{\Delta}^{2}}\left[4 \tau+\left(\mu_{\Delta}+1\right)^{2}\right]^{2}\left[4 \tau+\left(\mu_{\Delta}-1\right)^{2}\right] C_{3}^{V 2}, \\
w_{2 V}=\left(\frac{\mathcal{T}^{2}}{3}\right) \frac{4 \tau}{\mu_{\Delta}^{2}}\left[4 \tau+\left(\mu_{\Delta}+1\right)^{2}\right] C_{3}^{V 2},
\end{gathered}
$$

for the vector contributions,

$$
\begin{aligned}
w_{1 A}= & \left(\frac{\mathcal{T}^{2}}{3}\right) \frac{1}{4}\left[4 \tau+\left(\mu_{\Delta}+1\right)^{2}\right] \\
& \times\left[\left(4 \tau-\mu_{\Delta}^{2}+1\right) C_{4}^{A}-2 C_{5}^{A}\right]^{2}, \\
w_{2 A}=\left(\frac{\mathcal{T}^{2}}{3}\right) & \frac{1}{\mu_{\Delta}^{2}}\left[4 \tau+\left(\mu_{\Delta}+1\right)^{2}\right]\left(4 \tau \mu_{\Delta}^{2} C_{4}^{A 2}+C_{5}^{A 2}\right),
\end{aligned}
$$

$$
\begin{aligned}
u_{1 A}= & -\left(\frac{\mathcal{T}^{2}}{3}\right) \frac{1}{16 \tau \mu_{\Delta}^{2}}\left[4 \tau+\left(\mu_{\Delta}+1\right)^{2}\right]\left(C_{5}^{A}-4 \tau C_{6}^{A}\right) \\
& \times\left\{\left[48 \tau^{2}-\left(\mu_{\Delta}^{2}-1\right)^{2}+8 \tau\left(\mu_{\Delta}^{2}+1\right)\right] C_{5}^{A}\right. \\
+ & 4 \tau\left[4 \mu_{\Delta}^{2}\left(4 \tau+\mu_{\Delta}^{2}-1\right) C_{4}^{A}-\left[16 \tau^{2}+\left(\mu_{\Delta}^{2}-1\right)^{2}\right.\right. \\
+ & \left.\left.\left.8 \tau\left(\mu_{\Delta}^{2}+1\right)\right] C_{6}^{A}\right]\right\}, \\
u_{2 A}= & \left(\frac{\mathcal{T}^{2}}{3}\right) \frac{1}{4 \tau \mu_{\Delta}^{2}}\left[4 \tau+\left(\mu_{\Delta}+1\right)^{2}\right]\left(C_{5}^{A}-4 \tau C_{6}^{A}\right) \\
& \times\left[8 \tau \mu_{\Delta}^{2} C_{4}^{A}+\left(4 \tau-\mu_{\Delta}^{2}+1\right) C_{5}^{A}\right],
\end{aligned}
$$

for the axial-vector contributions; and

$$
\begin{aligned}
w_{3}= & \left(\frac{\mathcal{T}^{2}}{3}\right) \frac{C_{3}^{V}}{\mu_{\Delta}}\left[4 \tau+\left(\mu_{\Delta}+1\right)^{2}\right] \\
& \times\left[2 C_{5}^{A}-\left(4 \tau-\mu_{\Delta}^{2}+1\right) C_{4}^{A}\right]
\end{aligned}
$$

for the $V / A$ interference.

One finds that $u_{1 A}$ and $u_{2 A}$ (which arise from PCAC) are negligible, whereas the other functions are all significant. The latter are seen to fall strongly with increasing $q$. 
[1] W. M. Alberico, A. Molinari, T. W. Donnelly, E. L. Kronenberg, and J. W. Van Orden, Phys. Rev. C 38, 1801 (1988).

[2] D. B. Day, J. S. McCarthy, T. W. Donnelly, and I. Sick, Ann. Rev. Nucl. Part. Sci. 40, 357 (1990).

[3] T. W. Donnelly and I. Sick, Phys. Rev. Lett. 82, 3212 (1999).

[4] T. W. Donnelly and I. Sick, Phys. Rev. C 60, 065502 (1999).

[5] C. Maieron, T. W. Donnelly, and I. Sick, Phys. Rev. C 65, 025502 (2002).

[6] M. B. Barbaro, J. A. Caballero, T. W. Donnelly, and C. Maieron, Phys. Rev. C 69, 035502 (2004).

[7] M. B. Barbaro, R. Cenni, A. De Pace, T. W. Donnelly, and A. Molinari, Nucl. Phys. A643, 137 (1998).

[8] J. E. Amaro, M. B. Barbaro, J. A. Caballero, T. W. Donnelly, and A. Molinari, Phys. Rep. 368, 317 (2002).

[9] J. E. Amaro, M. B. Barbaro, J. A. Caballero, T. W. Donnelly, and A. Molinari, Nucl. Phys. A697 388 (2002).

[10] J. E. Amaro, M. B. Barbaro, J. A. Caballero, T. W. Donnelly, and A. Molinari, Nucl. Phys. A723, 181 (2003)

[11] Y. Fukuda et al. [Super-Kamiokande Collaboration], Phys. Rev. Lett. 81, 1562 (1998); M. H. Ahn et al. [K2K Collaboration], Phys. Rev. Lett. 90 041801, (2003).

[12] E. Ables et al. [MINOS Collaboration], A long baseline neutrino oscillation experiment at Fermilab, FERMILAB-Proposal0875 .

[13] E. Church et al. [BooNE Collaboration], A proposal for an experiment to measure muon-neutrino to electron-neutrino oscillations and muon-neutrino disappearance at the Fermilab Booster: BooNE, Fermilab-Proposal-0898.

[14] I. Ambats et al. [NOvA Collaboration], NOvA proposal to build an off-axis detector to study muon-neutrino to electron-neutrino oscillations in the NuMI, Fermilab-Proposal-0929.

[15] D. Drakoulakos et al. [MINERvA Collaboration], Proposal to perform a high-statistics neutrino scattering experiment using a fine-grained detector in the NuMI beam, hep-ex/0405002.

[16] C. J. Horowitz, H. C. Kim, D. P. Murdock, and S. Pollock, Phys. Rev. C 48, 3078 (1993).

[17] H. Kim, J. Pieckarewicz, and C. J. Horowitz, Phys. Rev. C 51, 2739 (1995).

[18] M. B. Barbaro, A. De Pace, T. W. Donnelly, A. Molinari, and M. J. Musolf, Phys. Rev. C 54, 1954 (1996).
[19] J. E. Amaro, J. A. Caballero, T. W. Donnelly, A. M. Lallena, E. Moya de Guerra, and J. M. Udias, Nucl. Phys. A602, 263 (1996).

[20] H. Kim, S. Schramm, and C. J. Horowitz, Phys. Rev. C 53, 2468 (1996).

[21] W. M. Alberico et al., Nucl. Phys. A623, 471 (1997); Phys. Lett. B438, 9 (1998); Nucl. Phys. A651, 277 (1999).

[22] L. Alvarez-Ruso, S. K. Singh, and M. J. Vicente Vacas, Phys. Rev. C 59, 3386 (1999).

[23] C. Maieron, M. C. Martinez, J. A. Caballero, and J. M. Udias, Phys. Rev. C 68, 048501 (2003).

[24] C. Bleve et al., Astropart. Phys. 16, 145 (2001).

[25] A. Meucci, C. Giusti, and F. D. Pacati, Nucl. Phys. A739, 277 (2004).

[26] J. Nieves, J. E. Amaro, and M. Valverde, Phys. Rev. C 70, 055503 (2004); nucl-th/0408005.

[27] J. E. Amaro, C. Maieron, J. Nieves, and M. Valverde (submitted to Nucl. Phys. A); nucl-th/0409017.

[28] R. Cenni, T. W. Donnelly, and A. Molinari, Phys. Rev. C 56, 276 (1977).

[29] J. E. Amaro, M. B. Barbaro, J. A. Caballero, T. W. Donnelly, and A. Molinari, Nucl. Phys. A643, 349 (1998).

[30] A. De Pace, M. Nardi, W. M. Alberico, T. W. Donnelly, and A. Molinari, Nucl. Phys. A726, 303 (2003).

[31] A. De Pace, M. Nardi, W. M. Alberico, T. W. Donnelly, and A. Molinari, Nucl. Phys. A741, 249 (2004).

[32] J. Carlson, J. Jourdan, R. Schiavilla, and I. Sick, Phys. Rev. C 65, 024002 (2002).

[33] J. E. Amaro, M. B. Barbaro, J. A. Caballero, and F. K. Tabatabaei, Phys. Rev. C 68, 014604 (2003).

[34] J. E. Amaro, G. Co, and A. M. Lallena, Nucl. Phys. A578, 365 (1994).

[35] J. Jourdan, Nucl. Phys. A603, 117 (1996).

[36] G. Höhler et al., Nucl. Phys. B114, 505 (1976).

[37] P. Amore, R. Cenni, T. W. Donnelly, and A. Molinari, Nucl. Phys. A615, 353 (1997).

[38] J. E. Amaro, M. B. Barbaro, J. A. Caballero, T. W. Donnelly, and A. Molinari, Nucl. Phys. A657, 161 (1999).

[39] T. W. Donnelly and R. D. Peccei, Phys. Rep. 50, 1 (1979).

[40] T. W. Donnelly, Prog. Part. Nucl. Phys. 13, 183 (1985).

[41] T. deForest and J. D. Walecka, Adv. in Phys. 15, 1 (1966).

[42] T. W. Donnelly and A. S. Raskin, Annals Phys. 169, 247 (1986). 